\title{
CHEERS Results from NGC 3393. III. Chandra X-Ray Spectroscopy of the Narrow Line Region
}

\author{
W. Peter Maksym ${ }^{1}$ (i) , Giuseppina Fabbiano ${ }^{1}$ (D), Martin Elvis ${ }^{1}$ (i), Margarita Karovska ${ }^{1}$ (D), Alessandro Paggi ${ }^{1,2,3,4}$ (D), \\ John Raymond $^{1}$ (D), Junfeng Wang ${ }^{5}$ (D), Thaisa Storchi-Bergmann ${ }^{6}$ (D), and Guido Risaliti ${ }^{7}$ (D) \\ ${ }^{1}$ Harvard-Smithsonian Center for Astrophysics, 60 Garden S., Cambridge, MA 02138, USA; walter.maksym@cfa.harvard.edu \\ ${ }^{2}$ Dipartimento di Fisica, Universit degli Studi di Torino, via Pietro Giuria 1, I-10125 Torino, Italy \\ ${ }^{3}$ Istituto Nazionale di Fisica Nucleare, Sezione di Torino, via Pietro Giuria 1, I-10125 Torino, Italy \\ ${ }^{4}$ INAF-Osservatorio Astrofisico di Torino, via Osservatorio 20, I-10025 Pino Torinese, Italy \\ ${ }^{5}$ Department of Astronomy, Physics Building, Xiamen University Xiamen, Fujian, 361005, People's Republic of China \\ ${ }^{6}$ Departamento de Astronomia, Universidade Federal do Rio Grande do Sul, IF, CP 15051, 91501-970 Porto Alegre, RS, Brazil \\ 7 INAF-Arcetri Astrophysical Observatory, Largo E. Fermi 5, I-50125 Firenze, Italy \\ Received 2018 October 30; accepted 2018 November 28; published 2019 February 13
}

\begin{abstract}
We present spatially resolved Chandra narrow-band imaging and imaging spectroscopy of NGC 3393. This galaxy hosts a Compton-thick Seyfert 2 active galactic nucleus (AGN) with sub-kpc bipolar outflows that are strongly interacting with the circumnuclear gas. We identify narrow-band excess emission associated with the Ne IX $0.905 \mathrm{keV}$ transition (with likely contributions due to intermediate-state iron emission) that points to strong shocks driven by AGN feedback. Imaging spectroscopy resolves outflow-interstellar medium (ISM) interaction sites and the surrounding ISM at $\sim 100 \mathrm{pc}$ scales, and suggests the presence of a hot AGN wind above the plane at radii beyond the shock sites. The cross-cone shows evidence for reprocessing of photoionization that has passed through gaps in the torus, and also for collisionally excited plasma that may be powered by a shock-confined equatorial outflow. Deep X-ray observations at subarcsecond resolution (such as may be performed very efficiently by Lynx, which would also energetically resolve the complex line emission) are necessary to eliminate model degeneracies and reduce uncertainties in local feedback properties.
\end{abstract}

Key words: galaxies: active - galaxies: jets - galaxies: individual (NGC 3393) - galaxies: Seyfert - X-rays: galaxies

\section{Introduction}

The ubiquity of supermassive black holes (SMBHs) in galactic nuclei does not appear to arise from coincidence. Rather, SMBHs and their host galaxies appear to form and coevolve, with the properties and behavior of each informing the other (Heckman \& Best 2014). In particular, rapidly accreting active galactic nuclei (AGNs) produce photoionizing radiation, jets, and winds that can modulate star formation in the host galaxy and accretion by the SMBH (Fabian 2012; Gaspari \& Sadowski 2017) via feedback processes, which must be considered in addition to those seen in rapid starbursts (Dekel \& Silk 1986; Silk 1997).

Although AGN feedback processes are easily observed on large scales in clusters, groups, and massive galaxies (Voit et al. 2015), statistical studies of SMBH obscuration show that radiative AGN feedback shapes galactic nuclei down to parsec scales Ricci et al. (2017). The low-density gas of the AGN extended narrow line region (ENLR) is sensitive to both kinematic outflows and radiative excitation associated with feedback, making studies of the ENLR particularly valuable.

Via the CHEERS (CHandra Extended Emission Line Region Survey) program, we are investigating the X-ray signatures of AGN feedback in nearby Seyfert galaxies. By spatially resolving X-ray emission within the ENLR, we can directly measure radiation from gas that has been collisionally ionized by AGN outflows or which arises from reprocessed photoionizing emission (Fabbiano et al. 2017, 2018a, 2018b; Paggi et al. 2017). The velocities $\left(v \sim 1000 \mathrm{~km} \mathrm{~s}^{-1}\right)$ of AGN ENLR outflows lead to characteristic energies of AGN feedback ( $k T \sim 1 \mathrm{keV})$, making X-rays the appropriate band for such measurements; in addition, measurement of multiple high ionization species in emission can be used to break degeneracies between models dominated by photoionized reflection or collisionally ionized plasma.

X-ray studies of systems with outflows smaller than $\sim$ few kpc (Wang et al. 2011a, 2011b, 2011c; Paggi et al. 2012; Bogdán et al. 2017; Fabbiano et al. 2018a) have shown that the ENLR in these systems is not a single photoionized plasma but rather a multiphase gas where photoionized emission may coexist simultaneously with contributions from collisionally ionized plasma stimulated by interactions between the outflows and the ENLR.

This is the third paper in our series of CHEERS papers on NGC 3393, (we refer to Maksym et al. 2016, 2017, as "Paper I" and "Paper II," respectively). NGC 3393 is a nearby $(D=53$ Mpc) barred spiral galaxy hosting a Seyfert 2 active nucleus (Diaz et al. 1988; de Vaucouleurs et al. 1991). The AGN is Compton-thick (Maiolino et al. 1998; Guainazzi et al. 2005; Burlon et al. 2011; Koss et al. 2015), so extended soft X-ray emission emitted by the nuclear interstellar medium (ISM) can be spatially resolved without being dominated by the AGN accretion disk or corona. Short Chandra observations (Bianchi et al. 2006; Levenson et al. 2006) showed extended X-ray emission associated with biconical optical [O III] emission. Fabbiano et al. (2011) claimed that deeper CHEERS revealed double hard point sources, indicating a binary AGN with subarcsecond separation, though this result was challenged by Koss et al. (2015). Koss et al. (2015) did confirm the association between the narrow line region (NLR) and soft X-rays using the CHEERS observations and zeroth-order Chandra imaging.

Continuum-subtracted narrow line observations using the Hubble Space Telescope (HST) show that while the ENLR of 
Table 1

Chandra ACIS-S Imaging Observations of NGC 3393

\begin{tabular}{lcc}
\hline \hline Obsid & Date & ks \\
\hline 04868 & 2004 Feb 28 & 29.33 \\
12290 & 2011 Mar 12 & 69.16 \\
\hline Total & $\ldots$ & 98.49 \\
\hline
\end{tabular}

the nuclear bicone is Seyfert-like, the surrounding medium forms a Low Ionization Nuclear Emission Region (LINER) cocoon (Paper I). Subsequent analysis in Paper II shows that while the LINER could be caused by photoionization filtered by the bicone gas or by slow shocks from lateral expansion of a biconical outflow, fast (FWHM $\gtrsim 1000 \mathrm{~km} \mathrm{~s}^{-1}$ Fischer et al. 2013) photoionizing shocks (as proposed by Cooke et al. 2000) cannot be excluded as a contributor to excitation within the bicone. This picture is supported by [O III] and $\mathrm{H} \alpha$ kinematics at sites of outflow-ISM interaction with enhanced X-ray emission. Fast shocks are an alternative to the suggestion by Koss et al. (2015) that emission at $1.8 \mathrm{keV}$ (consistent with e.g., $\mathrm{Mg}$ XII and Si XIII) indicates emission photoionized by the AGN.

We have investigated the spatially resolved spectroscopic properties of the X-ray emission associated with these processes. Chandra's subarcsecond angular resolution is critical for this analysis due to the complex and compact morphology of the system. We find evidence for simultaneous contributions from reprocessing of photoionizing radiation and collisional feedback-driven plasma throughout the nucleus (including in the cross-cone, pointing to gaps in the torus, e.g., Paggi et al. 2012; Fabbiano et al. 2018a). In particular, we find evidence for shocks as significant contributors to the extended $\mathrm{X}$-ray emission. The physical properties of that plasma indicate that the interactions between the AGN and gas in the inner galactic plane may be sufficient to unbind gas from the host galaxy, but it is not clear that this is the case (as suggested by evidence for trapped hot plasma that may be collisionally excited by a shock-confined equatorial outflow).

Throughout this paper, we adopt concordant cosmological parameters $^{8}$ of $H_{0}=70 \mathrm{~km}^{-1} \mathrm{~s}^{-1} \mathrm{Mpc}^{-1}, \Omega_{m, 0}=0.3$ and $\Omega_{\Lambda, 0}=$ 0.7. All coordinates are $\mathrm{J} 2000$. In all figures, celestial North is up and color scales are logarithmic (unless otherwise noted). For distance evaluation we use the Theureau et al. (1998) determination of redshift $z=0.0125$ from observations of the $21 \mathrm{~cm}$ neutral hydrogen emission line, such that NGC 3393 is at distance $D=53 \mathrm{Mpc}$ with linear scale $257 \mathrm{pc} \mathrm{arcsec}^{-1}$. The redshift corresponds to $7 \mathrm{eV}$ for a photon emitted at $600 \mathrm{eV}$, which is negligible compared to the $\sim 100 \mathrm{eV} \mathrm{FWHM}$ of ACIS$\mathrm{S}$, so redshift corrections are negligible.

\section{Observations and Data Reduction}

We have obtained two observations totaling $\sim 100 \mathrm{ks}$ of ACIS-S exposure from the Chandra Data Archive9 (Table 1). Since the Chandra gratings may introduce uncalibrated distortions to the point spread function (PSF) on subarcsecond scales, ${ }^{10}$ we do not use zeroth-order grating images. As in

\footnotetext{
Distances are calculated according to http://www.astro.ucla.edu/ wright/ CosmoCalc.html.

9 http://cda.harvard.edu/chaser

${ }^{10}$ For a discussion of the Chandra zeroth order, see http://asc.harvard.edu/ proposer/POG/html/HETG.html.
}

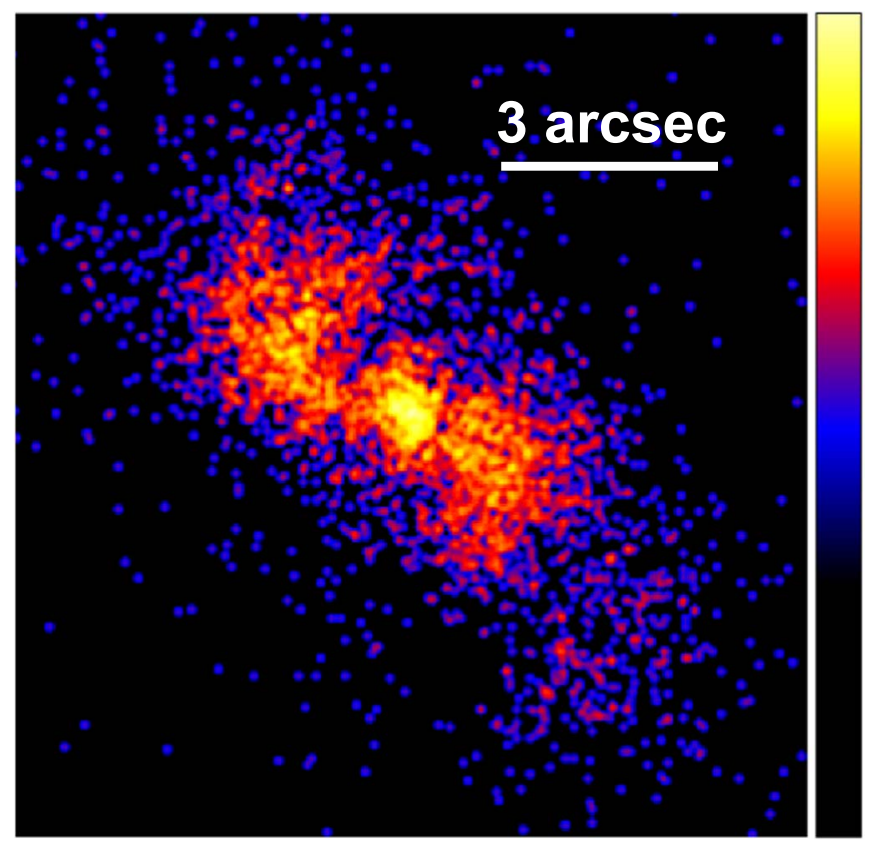

Figure 1. Chandra $0.3-2 \mathrm{keV}$ image of the NGC 3393 nucleus. Photons are binned to $1 / 16$ th ACIS pixel size and smoothed with a Gaussian where $r=3$ pixels.

Paper II, we reprocessed the data with the Chandra Interactive Analysis of Observations package (CIAO; Fruscione et al. 2006) using sub pixel event positioning, removing periods of strong background flaring, and merging data from both obsids (with merge_obs) to produce an event file equivalent to a single long exposure. We describe the use of other data reduction techniques as appropriate in subsequent subsections. We generated all radial profile maps for X-ray, optical, and radio data using the CIAO tool dmextract.

Data processing and production of continuum-subtracted [O III] $\lambda 5007 \AA$ images for HST WFC3 data are also described in detail in Paper II.

\subsection{X-Ray Imaging and Spectral Features}

\subsubsection{X-Ray Continuum Emission}

In Paper II, we investigated the broadband X-ray emission of the NGC 3393 nucleus (Figure 1). The brightest part of the soft emission is located in the nucleus (where there is a central radio peak), and wraps around the radio lobes in an S-shape. We observed that the [O III] and soft X-rays are effectively cospatial (as in Levenson et al. 2006; Koss et al. 2015), but find a fuller picture using deconvolved broadband $(0.3-8 \mathrm{keV})$ maps, optical narrow line emission maps, and other multiwavelength data. Namely, we inferred that although the bulk of the X-ray emission is likely photoionized, shock emission may lead the radio lobes, particularly given that the shocks are likely to be fast enough to be photoionizing. We also identified extended hard (2-8 keV) emission that traces the brightest parts of the continuum emission.

\subsubsection{X-Ray Emission Line Maps}

We begin our X-ray spectral analysis by investigating the morphological distribution of X-ray emission of known ion species previously studied by Wang et al. (2011a) and Paggi et al. (2012). 
In each band, we produced merged event files, and images with both $1 / 8$ pixel and $1 / 4$ pixel $(0$ " 062,0 " 123$)$ spatial bins. We generated exposure maps and fluxed images for the combined data set using the CIAO script merge_obs.

In Figure 2, we map photons in bands of varying width covering the $\mathrm{O}$ VII triplet at $\sim 0.569 \mathrm{keV}$, the $\mathrm{O}$ VIII Ly $\alpha$ transition at $0.654 \mathrm{keV}$, and the Ne IX triplet at $\sim 0.915 \mathrm{keV}$. Given the ACIS-S energy resolution of $\sim 100 \mathrm{eV},{ }^{11}$ we define these bands such that in the observer frame, $0.526 \mathrm{keV}<\mathrm{O} \mathrm{VII}<$ $0.626 \mathrm{keV}, 0.624 \mathrm{keV}<\mathrm{O}$ VIII $<0.674 \mathrm{keV}$, and $0.880 \mathrm{keV}<$ $\mathrm{Ne}$ IX $<0.954 \mathrm{keV}$.

Figure 2 shows two columns with O VII (a, b), O VIII (c, d), and Ne IX (e, f) images overlaid with the [O III] (right) and $8 \mathrm{GHz}$ radio continuum (left) as white contours.

According to Figure 2, the overall distribution of photons in each X-ray band follows similar trends to those of the deconvolved broadband $(0.3-8 \mathrm{keV})$ maps described in Paper II: the X-rays generally follow the [O III] maps, and avoid the bubbles corresponding to VLA $8.46 \mathrm{GHz}$ emission. We identify noticeable differences between the species, however:

1. O VII appears to best trace the low-intensity extended [O III] contour, while Ne IX is associated with narrow, high-intensity [O III] arcs that wrap around the radio outflows.

2. O VIII does not have an obvious S-shape. Rather, O VIII appears linear, narrowly confined to a $D \sim 1^{\prime \prime}$ cylinder oriented NE-SW along the bicone. There may be a loop to the NE, but there are too few photons to test its putative extent.

3. Within $\sim 1^{\prime \prime}$ of the nucleus, O VII forms a bright E-W bar that is associated with a similar bar in [O III]; this bar is not seen in O VIII and Ne IX.

4. The brightest Ne IX-emitting regions are in closer contact with the radio outflows, whereas $\mathrm{O}$ VII is more extended (Figure 2, (f)).

The narrow, linear shape of O VIII appears simple, so that more rigorous investigation of faint sub-structure is not warranted for this data set. But the identification of more complex structural differences between O VII and Ne IX on subpixel scales may be unreliable due to the low surface brightness of the S-shaped arms in X-rays (within $r<3^{\prime \prime}$ from the nucleus, we find mean surface brightness $\bar{I} \sim 0.73$ count $\operatorname{arcsec}^{-2}$ in $\mathrm{O}$ VII). We therefore tested for the presence of these line emission features via adaptive smoothing, hardness ratio maps, and radial profile binning.

\subsubsection{Adaptive Smoothing}

In order to test the veracity of differing line emission morphologies identified in Section 2.1.2, we first created adaptively smoothed images of Ne IX and O VII in the inner $r<10^{\prime \prime}$ of NGC 3393. We used the csmooth tool from CIAO to create images at $1 / 8$ native ACIS pixel scale, with minimum feature significance of $2 \sigma$ and maximum significance of $5 \sigma$. The 1/8 pixel scale map is displayed in Figure 3.

The enhanced Ne IX is clearly cospatial with the strongest emission in the [O III] arcs (not shown, but see Section 2.1.4 and Figure 4) leading the radio knots, whereas O VII traces the central [O III] bar and the weaker [O III] clouds at larger radii (as shown

\footnotetext{
${ }_{11}$ Chandra Proposer's Guide; http://cxc.harvard.edu/proposer/POG/html/ chap6.html.
}

in Figure 2(b). There is some overlap between the strongest Ne IX and O VII in the SW bicone, but in the NE peak emission from the the two lines is physically separate at $d \gtrsim 0$ "' 5 .

\subsubsection{Hardness Ratio Mapping}

In order to investigate the relative importance of the Ne IX and O VII features identified in Section 2.1.3, we created a hardness ratio map of the adaptively smoothed images in Figure 4, such that $\mathrm{HR}=(\mathrm{H}-\mathrm{S}) /(\mathrm{H}+\mathrm{S})$, where the hard band $\mathrm{H}$ is Ne IX and soft band $S$ is O VII. [O III] contours are overlaid for comparison. This hardness ratio map shows the same patterns described in Figure 3 and Section 2.1.3.

As a second test of the significance of the Ne IX and O VII features identified in Section 2.1.2, we defined several spatial bins based upon apparent peaks and dips in the Ne IX/O VII map. We then calculated hardness ratios in each of these bins using Bayesian Estimation of Hardness Ratios (BEHR; Park et al. 2006), which is well suited to quantifying hardness ratio uncertainties in the regime of Poisson statistics. The hardness ratios of each region are depicted in Figure 4 (right), and listed in Table 2. The Ne IX/O VII structural patterns display typical differences of $\lesssim 2 \sigma$ in Ne IX-O VII hardness ratio from the region with weakest Ne IX/O VII (region 13), but regions 8, 9, 10,11 , and 12 all differ by $\gtrsim 3 \sigma$ from region 13 . Region 13 is within the bicone but not closely adjacent to the brightest radio knots, whereas all high-Ne IX/O VII regions are directly adjacent to bright radio knots. Regions 4 and 5 cover the $\mathrm{E}-\mathrm{W}$ bar of strong $\mathrm{O}$ VII.

\subsubsection{Soft X-Ray Emission and [O III]}

In order to investigate the commonly observed relationship between soft (0.3-2 keV) X-ray emission and [O III], we generated radial surface brightness profiles in each band with 0 ". 25 annular bins (switching to $1^{\prime \prime}$ bins at $r \sim 5^{\prime \prime}$ due to the presence of low surface brightness regions). We used the CIAO tool dmextract to generate the profiles. We used the WFC3 header parameters PHOTFLAM and PHOTBW to calculate [O III] fluxes, ${ }^{12}$ converting rms bandwidth to FWHM bandwidth. For the soft X-ray flux we assumed the median in-band (0.3-2 keV) photon energy at $r<3^{\prime \prime}(E=0.911 \mathrm{keV})$.

We show the $[\mathrm{O} \mathrm{III]} /(0.3-2 \mathrm{keV})$ ratio profile in Figure 5, and indicate peaks of the radio emission with brown vertical lines. We observe that the ratio is flat at $r \gtrsim 6^{\prime \prime}$, but spikes sharply just outside the radio lobes $(r \sim 1$ ". 5$)$, and at $r \sim 5^{\prime \prime}$ in the SW cone (but not the NE). We see $[\mathrm{O} \mathrm{III]} /(0.3-2 \mathrm{keV})$ ratio troughs at the nucleus and immediately outside the [O III] / $(0.3-2 \mathrm{keV})$ spikes, which are associated with the radio lobes (at $r \sim 3^{\prime \prime}-4^{\prime \prime}(\mathrm{NE})$ and $r \sim 2 . ! 5(\mathrm{SW})$.

In order to investigate the $[\mathrm{O} \mathrm{III]} /(0.3-2 \mathrm{keV})$ ratio independently of azimuthal effects, we have also generated a ratio map shown in Figure 6. We degraded the HST images with a $r=3$ - pixel Gaussian kernel. We then re-sampled the HST image to match 0.5 pixel $(0$ !'246) scale $0.3-2 \mathrm{keV}$ Chandra images using CIAO reproject_image. Finally, we used dmimgcalc to calculate the ratio map. We find that [O III]/ $(0.3-2 \mathrm{keV})$ trends are similar to the radial profile, but [O III]/ $(0.3-2 \mathrm{keV})$ is also elevated counter-clockwise to the radio emission. For $r \lesssim 3^{\prime \prime}$, X-rays are suppressed in the cross-cone and interior to the radio lobes.

\footnotetext{
12 http://www.stsci.edu/hst/wfc3/documents/handbooks/currentDHB/ Chapter2_data_structure5.html
} 

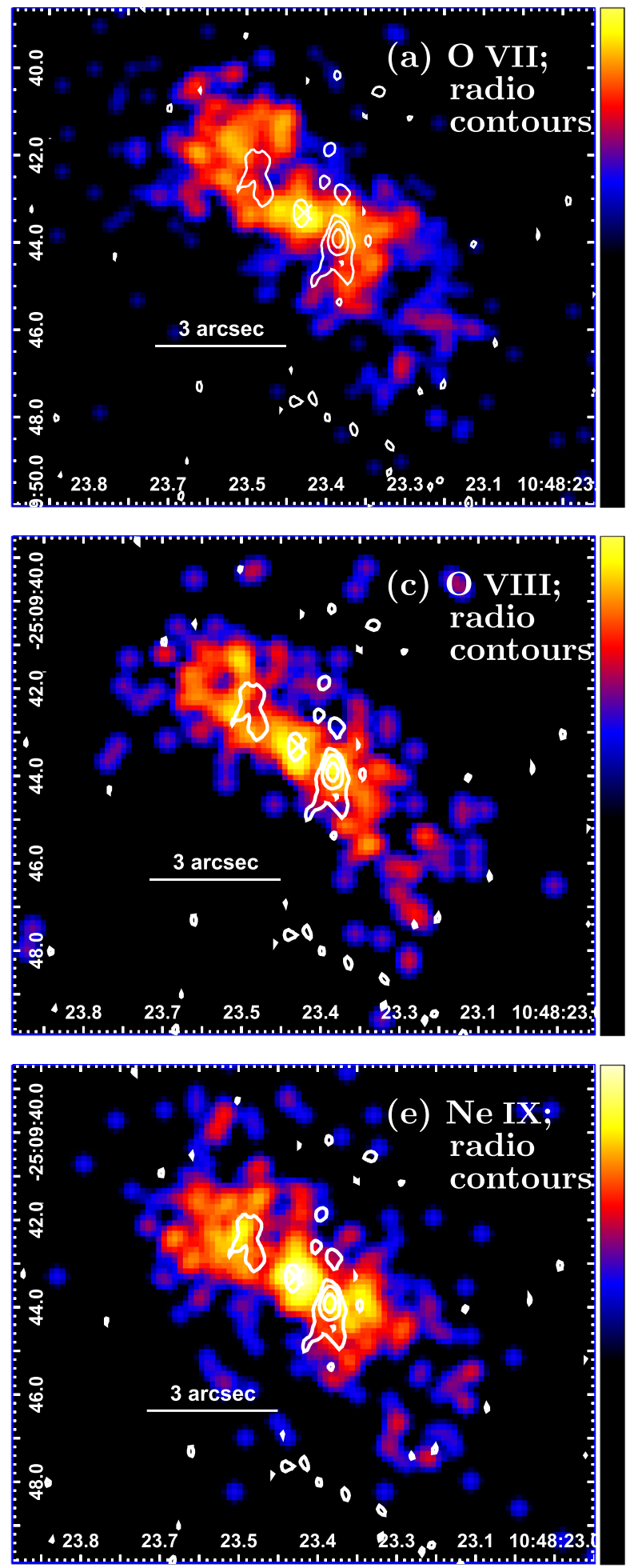
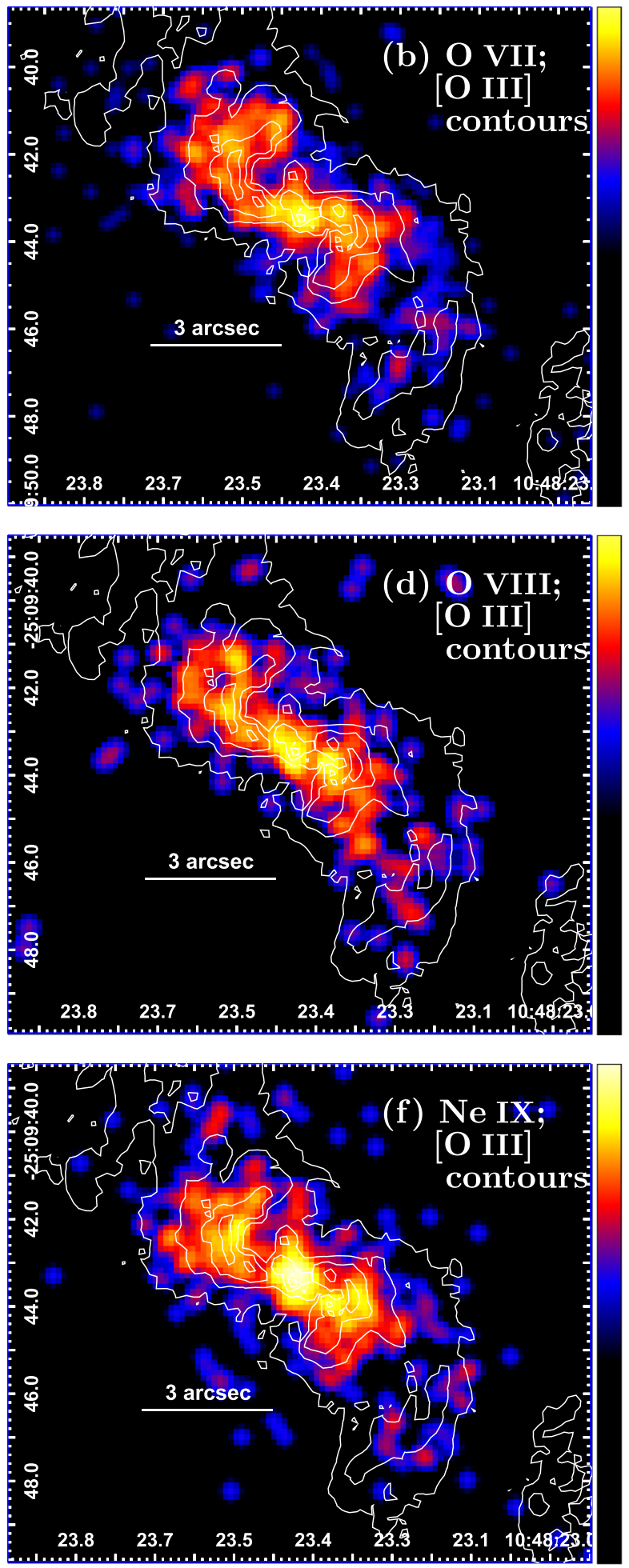

Figure 2. X-ray emission line maps from Chandra observations of NGC 3393. The left column is overlaid with $8.46 \mathrm{GHz}$ contours from Faint Images of the Radio Sky at Twenty-cm (FIRST) (as in Paper II) and the right column has contours from [O III] $\lambda 5007 \AA$, as observed by HST. From top to bottom, each row shows top: $\mathrm{O}$ VII triplet at $0.569 \mathrm{keV}$, center: the $\mathrm{O}$ VIII Ly $\alpha$ transition at $0.654 \mathrm{keV}$, and bottom: the Ne IX triplet at $0.915 \mathrm{keV}$. Photons are binned to 0 ." 123 scale, which is $1 / 4$ of the native ACIS pixel size, and smoothed with a 3 pixel Gaussian filter.

\subsubsection{X-Ray Emission Line Profiles}

In order to check the observed trends in the emission line ratios that were described in Sections 2.1.3 and 2.1.4 (particularly areas of elevated Ne IX/O VIII), we also binned emission line maps of Ne IX, O VIII, O VII, and $8.46 \mathrm{GHz}$ emission radially in conical profiles. These profiles cover the bicones in both the $\mathrm{NE}$ and $\mathrm{SW}$ with $105^{\circ}$ azimuthal extent. We plotted the resulting radial emission profiles in Figure 7 . We use $\Delta r=0$ ". 25 bins in order to approach mirror resolution, which is necessary for direct comparison to HST images. 


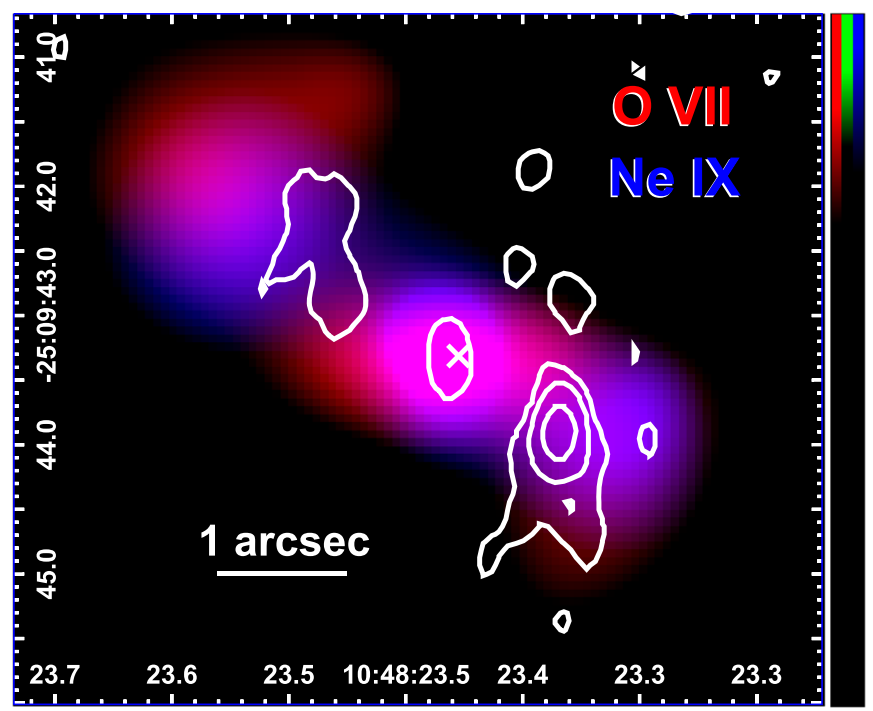

Figure 3. Adaptively smoothed emission map of Ne IX (blue) and O VII (red) with VLA $8.46 \mathrm{GHz}$ contours overlaid. High-significance emission features of these species are separated by $\sim 0$. $5-1^{\prime \prime}$ in the S-shaped arms, which precede the radio outflows. The E-W nuclear bar of O VII identified in Figure 2 remains prominent here.

Consistent with Section 2.1.3, we observe areas of multiple contiguous bins of increased Ne IX/O VII or O VIII/O VII. However, the significance is only $\sim 1 \sigma$ per bin.

In Figure 8, we use all three emission lines where both $\mathrm{O}$ VII and $\mathrm{O}$ VIII are related to Ne IX. Figure 8 (top) shows that there is a range in both $\mathrm{Ne}$ IX/O VII and $\mathrm{O}$ VIII/O VII, but no strong correlation (best-fit linear slope consistent with $\sim 1$ ). Several bins are outliers at the $1 \sigma-2 \sigma$ level, but the distribution of outliers is asymmetrical: all outliers have $\mathrm{Ne}$ IX $/ \mathrm{O}$ VII $\geqslant 1.0$, compared to typical values of 0.3-1.0 for the bins, which are consistent with the best fit. To illustrate how the outliers affect correlation, we find that the Spearman correlation coefficient is not significant for the full population ( $\rho=0.20$ with $\Delta \sigma=-0.98$ from the null hypothesis) but is modestly significant for $\mathrm{Ne} \mathrm{IX} / \mathrm{O}$ VII $<$ 1.0 ( $\rho=0.56$ with $\Delta \sigma=-2.19$ from the null hypothesis).

We see in Figure 8 (bottom) that a histogram of Ne IX/O VII shows a bimodal population divided at $\mathrm{Ne}$ IX $/ \mathrm{O}$ VII $=1.0$. The Ashman et al. (1994) D-statistic for the two populations is $D=4.9$, where $D>2$ is necessary for a clean separation.

To investigate the effects of coarser binning on the systematic trend in line ratios, we generated new line ratio radial profiles $\Delta r=1^{\prime \prime}$ bins and a narrower azimuthal extent that primarily covers an opening angle with bright Ne IX. This better emphasizes regions of elevated Ne IX/O VII. The signal-tonoise ratio $(\mathrm{S} / \mathrm{N})$ is improved (relative to the $r=2^{\prime \prime}-3^{\prime \prime}$ bins) for multiple regions of enhanced Ne IX/O VII and O VIII/O VII, but only at the $\sim 2.0-2.5$ level.

\subsection{Imaging Spectroscopy}

\subsubsection{Spectral Extraction and Statistics}

In order to model the hot X-ray emitting plasma in the nucleus of NGC 3393, we extracted spectra from various regions as extended sources using the CIAO tool specextract. These spectral regions include the extended emission within $r=5^{\prime \prime}$ of the nucleus, as well as various sub-regions defined in Section 2.1.4 to investigate Ne IX-O VII hardness ratios (see Figure 4).
The regions defined in Section 2.1.4 typically contain 150-1000 counts, such that Poisson statistics are relevant, particularly when attempting to take advantage of the ACIS energy resolution. In this regime, the use of $\chi^{2}$ for fit minimization can bias model parameters, and the required level of binning for valid $\chi^{2}$ fitting degrades the energy resolution of the spectrum. The Cash (1979) statistic is preferred for Poisson data, but does not permit background subtraction. Rather, it requires additional modeling of the background such as via a fit to the background region. To avoid this complexity, we used the L-statistic or "lstat" in Xspec (Arnaud 1996), which uses Bayesian analysis of Poisson data with Poisson background, and can be validly used with background subtraction. We grouped the $r \leqslant 5^{\prime \prime}$ spectrum at 1 count per energy bin, and all other spectra at 5 counts per energy bin. In our modeling, we use Xspec measurements of $\chi^{2}$ to illustrate goodness of fit.

\subsubsection{Spectral Modeling}

We expect harder emission near the nucleus to be at least partially contaminated by the wings of the point-spread function from the AGN, so we modeled an AGN component for all spectral fits based on Koss et al. (2015) fits of Nuclear Spectroscopic Telescope Array (NuSTAR) data. We extracted an AGN-dominated spectrum for $r \leqslant 0$." 4 from the nucleus, using the CIAO response correction for extended sources. Having observed extended X-ray emission up to $4 \mathrm{keV}$ (Maksym et al. 2017), we only fit energies above $4 \mathrm{keV}$. As per Koss et al. (2015), we included Gaussian Fe $\mathrm{K} \alpha$ emission (Xspec zgauss fixed at $6.4 \mathrm{keV}$ ), a warm absorber (wabs), absorbed and reflected power laws (plcabs and pexrav with $\Gamma=1.9, n H=3.1 \times 10^{24} \mathrm{~cm}^{-2}, k T_{\text {cutoff }}=200 \mathrm{keV}, \cos \theta_{i}=$ 0.45 and other parameters fixed to values in Koss et al. 2015). The final AGN component has only a single free normalization parameter, such that all sub-components are described in terms of that free normalization. We measured component flux via the convolution model cflux and determined parameter uncertainties via Monte Carlo Markov chain (MCMC) methods in Xspec.

The Composite ENLR-For the NLR component of the extended $r \leqslant 5^{\prime \prime}$ region, we begin by assuming a purely phenomenological model of Gaussian emission lines on a power-law continuum, comparable to Paggi et al. (2012) for Mrk 573 and Koss et al. (2015) for Chandra grating observations of NGC 3393 (which have superior energy resolution but worse counting statistics). We first assume a typical AGN continuum with fixed $\Gamma=1.8$ for the power-law index. When $\Gamma$ is left free, we find $\Gamma=3.1_{-0.13}^{+0.13}$ and an improved fit $\left(\chi^{2} /\right.$ dof $=1.18$ versus 1.41 for fixed $\left.\Gamma\right)$. The results of these fits are shown in Table 3 and Figure 9.

We explore more physically motivated models for the ENLR in NGC 3393 by fitting various combinations of model components used in Paggi et al. (2012) for Mrk 573. These include a collisionally ionized component (APEC; Smith et al. 2001), as well as a table model component constructed using CLOUDY (Ferland et al. 1998) by Paggi et al. (2012). This CLOUDY model describes photoionizing radiation reprocessed by diffuse gas under a grid of ionization parameters $(-2 \leqslant$ $\log U \leqslant 3)$ and column densities $\left(19.0 \leqslant \log N_{\mathrm{H}} \leqslant 23.5\right)$. Single-component fits are poor, so we explore two-component models, the results of which are indicated in Table 4 . The best two-component fit includes both thermal APEC and photoionized 

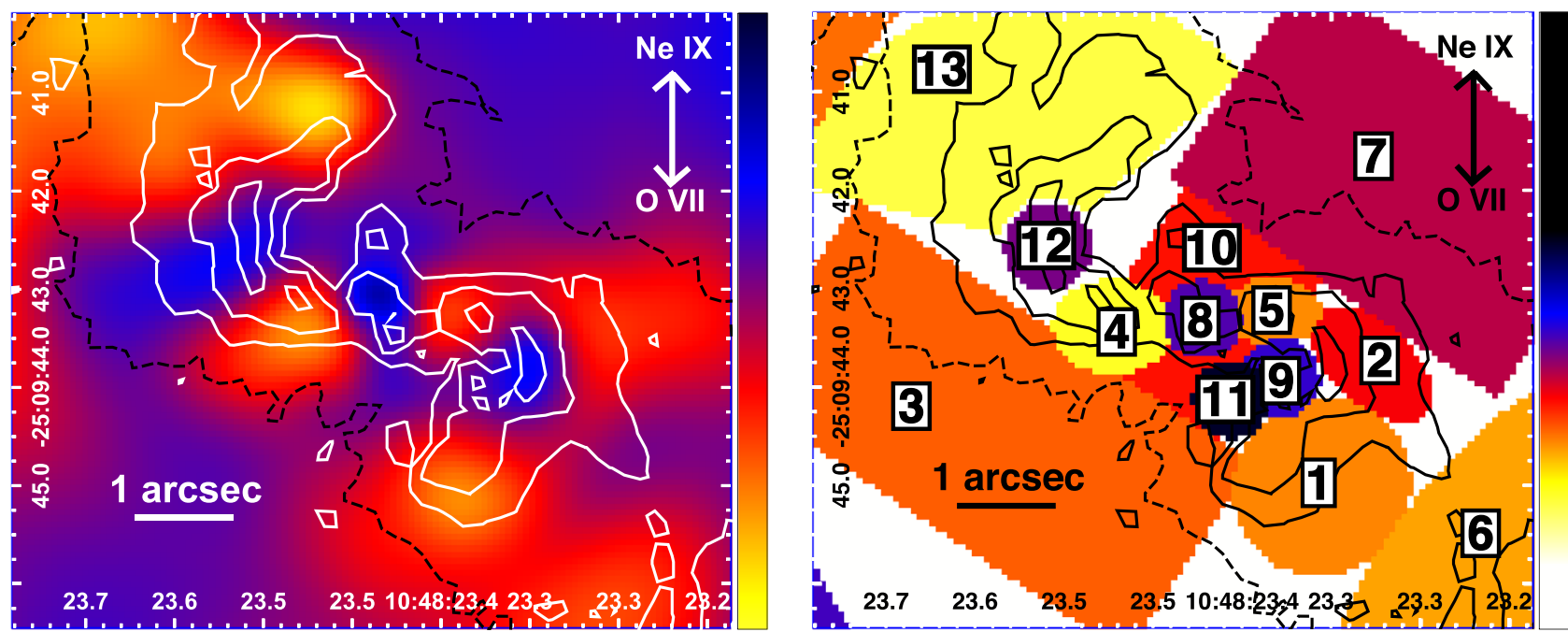

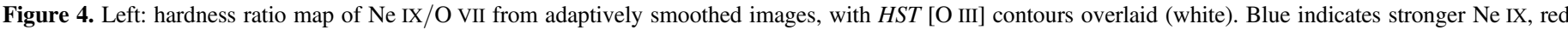

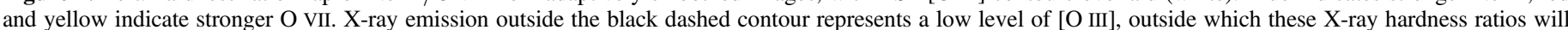

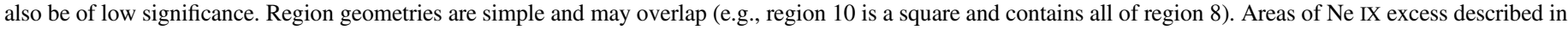

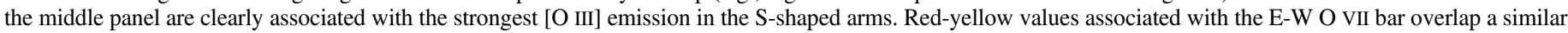

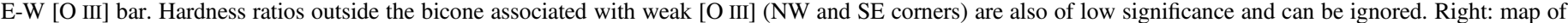

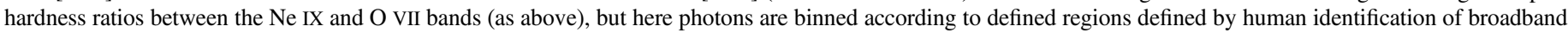

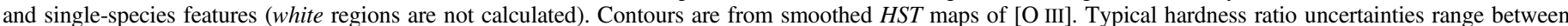
0.10 and 0.15 . These binned calculations confirm that Ne IX excesses identified in Figure 3 are statistically significant.

Table 2

Local Ne IX-O VII Hardness Ratios

\begin{tabular}{lc}
\hline \hline ID & HR \\
\hline 1 & $-0.39_{-0.12}^{+0.10}$ \\
2 & $-0.24_{-0.15}^{+0.15}$ \\
3 & $-0.35_{-0.14}^{+0.11}$ \\
4 & $-0.56_{-0.14}^{+0.09}$ \\
5 & $-0.40_{-0.16}^{+0.14}$ \\
6 & $-0.43_{-0.11}^{+0.09}$ \\
7 & $-0.19_{-0.16}^{+0.13}$ \\
8 & $-0.07_{-0.13}^{+0.12}$ \\
9 & $-0.03_{-0.19}^{+0.17}$ \\
10 & $-0.27_{-0.07}^{+0.07}$ \\
11 & $+0.25_{-0.26}^{+0.29}$ \\
12 & $-0.11_{-0.15}^{+0.14}$ \\
13 & $-0.60_{-0.06}^{+0.06}$ \\
\hline
\end{tabular}

Note. These are the mean BEHR-determined Bayesian values for $(\mathrm{Ne}$ IX-O VII $) /(\mathrm{Ne}$ IX $+\mathrm{O}$ VII $)$. ID values indicate the corresponding region numbers marked in Figure 4.

CLOUDY models in which a collisional APEC component $\left(k T=1.50_{-0.11}^{+0.18}\right)$ is an order of magnitude weaker than the photoionized component. This collisional component peaks near $\sim 1 \mathrm{keV}$ and is most significant relative to the CLOUDY model in a band containing complex emission by Ne IX, NeX, and Fe XXIV. When compared to the Gaussian fit, the slope of the continuum for the APEC+CLOUDY model is better represented by the model where $\Gamma$ is fit as a free parameter.

Spatially Resolving ENLR Spectra-Our previous analysis of spatial variation in spectral line emission suggests that physical conditions of the NGC 3393 ENLR vary significantly on scales of $\sim 10 \mathrm{~s}$ of $\mathrm{pc}$, in keeping with the analysis by Maksym et al. (2017). We therefore explore spatial variation of
Ionization Cones: Ratios

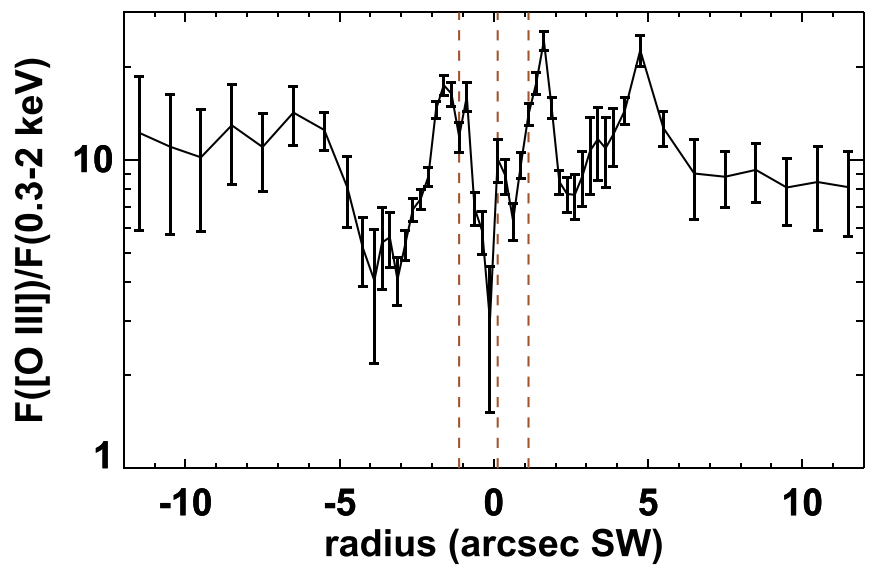

Figure 5. Radial profile of the ratio between $[\mathrm{O}$ III $] \lambda 5007 \AA$ emission and 0.3-2 keV X-ray flux. Brown vertical dashed lines indicate peaks of radio emission. We identify strong peaks immediately exterior to the radio peaks, as well as at $\sim 5^{\prime \prime} \mathrm{SW}$. The normalization is comparable values from Mrk 573 (Paggi et al. 2012) and NGC 4151 (Wang et al. 2011a) when those are corrected for bandwidth. Like Paggi et al. (2012) and NGC 4151 Wang et al. (2011a), we find spikes associated with high-density features like the leading edges of radio knots.

the $0.3-8 \mathrm{keV}$ spectra X-ray measured by Chandra by fitting the sub-regions in Section 2.1.4. The low numbers of counts limit the reliability of complex modeling on these spatial scales, so we explore single component APEC and CLOUDY models for the extended emission, as well as a combined APEC + CLOUDY model. The results of these fits are shown in Table 5 and Figure 11.

In general, single CLOUDY models are preferred to single APEC models, and the combined APEC+CLOUDY model is preferred in all cases except for region 7 (the NW cross-cone) where the measured APEC contribution is negligible. An excess of photons near $6.4 \mathrm{keV}$ raises the AGN component in 


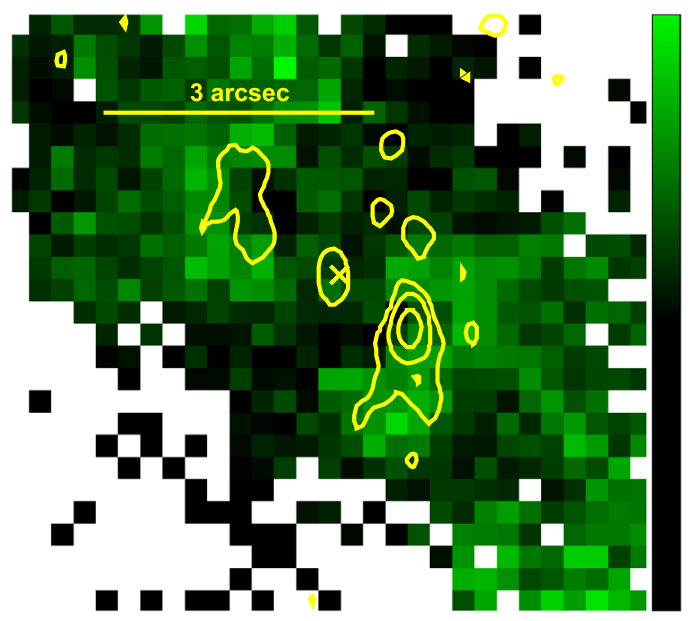

Figure 6. Ratio map of [O III] $]$ $5007 \AA$ emission to (0.3-2 keV) X-ray flux. HST resolution is degraded with a Gaussian kernel to match a Chandra photon image binned at 0.5 pixel $(0$ !' 246$)$ scale. Green indicates a high ratio. White pixels have zero X-ray photons and are hence undefined. Cyan contours are radio.

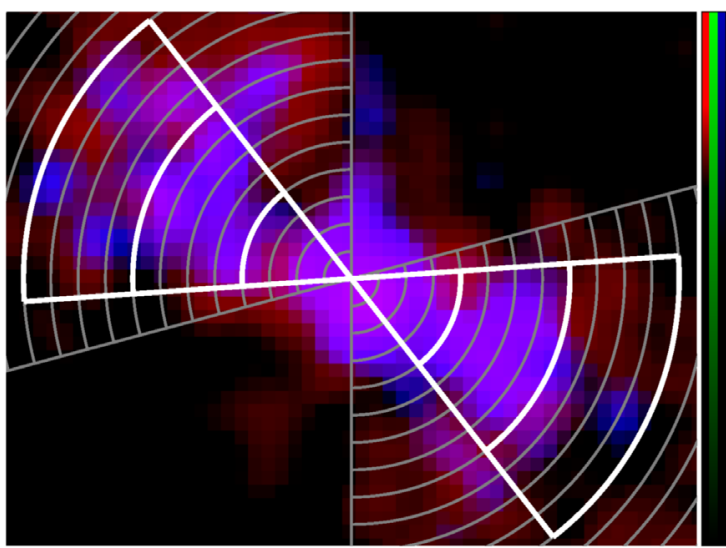

Ionization Cones: Ratios

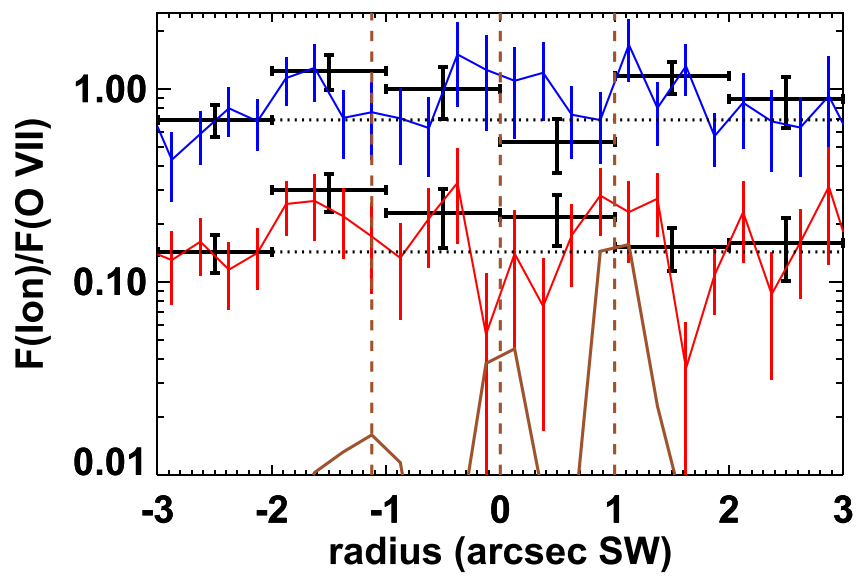

Figure 7. Top: emission line image of the inner $r<3^{\prime \prime}$ of NGC 3393, binned to 0 !" 25 with $r=2$ pixel Gaussian smoothing. Ne IX is blue and O VII is red. Annular arc contours represent different radial bins, extracted and plotted in the bottom panel. White contours have $\Delta r=1^{\prime \prime}$ spacing and a narrower angle, gray contours are broader, with $\Delta r=0$ ". 25 . Bottom: radial profiles of line ratios for the regions depicted above. For the $\Delta r=0$ !' 25 profile, Ne IX/O VII is blue and O VIII/O VII is red (with error bars in the same color). Black error bars are overlaid to show uncertainties for the $\Delta r=1^{\prime \prime}$ profile. Brown lines show radio emission for the same region, renormalized for ease of comparison, and the peaks of the three main radio blobs are indicated with vertical dashed lines. Horizontal dashed lines indicate line ratios at $-3<r<-2$ for ease of comparison.

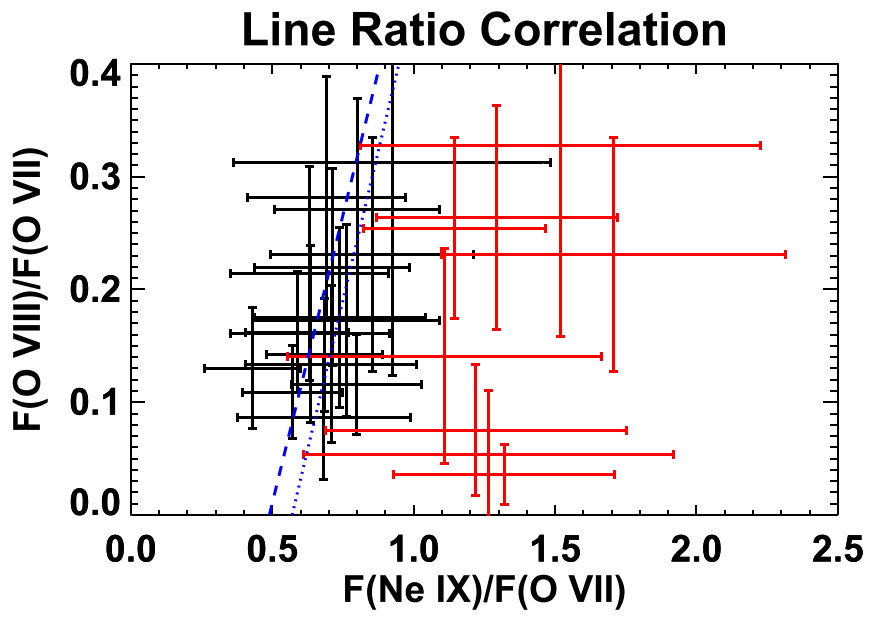

Ne IX - O VII Ratio Distribution

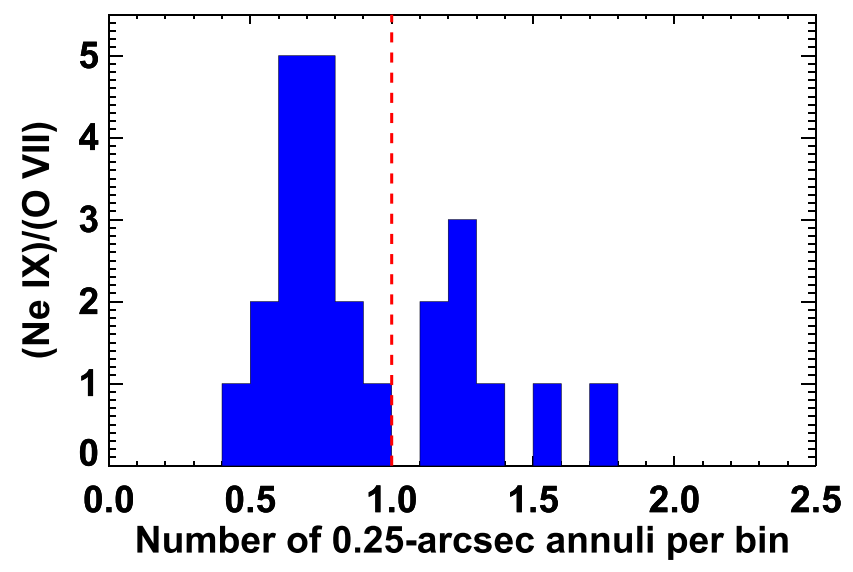

Figure 8. Top: O VIII/O VII from Figure $7(\Delta r=0$ ". 25 bins) plotted vs. $\mathrm{Ne}$ IX/O VII for correlation analysis. The best linear fit for the full data set (blue dotted line $)$ is dominated by low $(>1.0$, black) Ne IX/O VII values, as demonstrated by the small offset from the best fit (blue dashed line) that excludes high $(\geqslant 1.0$, red) outliers. Bottom: histogram of $\mathrm{Ne}$ IX/O VII values from above. The red dashed vertical line indicates Ne IX $/ \mathrm{O}$ VII $=1.0$, which marks the separation between black and red values in the top plot.

region 11 (a region in the $\mathrm{SW}$ cone with low surface brightness in X-rays, optical, and UV) such that residuals between 3 and $6 \mathrm{keV}$ become uniformly and significantly negative, so we introduce an additional Gaussian component fixed at $6.4 \mathrm{keV}$ (host frame) for region 11. Host column densities tend to be negligible, and fits with non-negligible column densities (such as the single APEC models of regions 3 and 6) tend to imply high component fluxes that are not consistent with the composite $r \leqslant 5^{\prime \prime}$ ENLR fit.

We caution that there is some degeneracy between different families of model solutions. For example, in some cases the difference in fit quality may be small between APEC + CLOUDY models with large $\log U$ and small $k T$, and models with small $\log U$ and large $k T$.

\section{Results}

In this paper, we show that the X-ray line emission morphology reveals structural complexity beyond what is possible with broadband imaging. This complexity goes beyond Paper II, where we already showed that the ENLR in NGC 3393 that although [O III] traces X-ray emission loosely (as expected from other studies), high-resolution deconvolved 
Table 3

Gaussian Composite Fits for $r<5^{\prime \prime}$

\begin{tabular}{|c|c|c|c|}
\hline & & Fixed $\Gamma$ & Free $\Gamma$ \\
\hline Species & $\begin{array}{c}\text { Rest } \\
\text { Energy (keV) }\end{array}$ & $\begin{array}{l}\text { Normalization } \\
\quad(1 E-07)\end{array}$ & $\begin{array}{l}\text { Normalization } \\
\quad(1 \mathrm{E}-07)\end{array}$ \\
\hline $\mathrm{C} \mathrm{V} \mathrm{He} \gamma$ & 0.371 & $540.82_{-132.36}^{+141.64}$ & $<63.85$ \\
\hline $\mathrm{N}$ VI triplet & 0.426 & $\ldots$ & $\ldots$ \\
\hline C IV Ly $\beta$ & 0.436 & $424.92_{-8.29}^{+80.36}$ & $135.05_{-116.56}^{+114.83}$ \\
\hline N VII Ly $\alpha$ & 0.500 & $290.51_{-46.82}^{+48.87}$ & $78.40_{-53.50}^{+53.70}$ \\
\hline O VII triplet & 0.569 & $732.31_{-51.90}^{+53.64}$ & $519.49_{-62.75}^{+63.59}$ \\
\hline O VIII Ly $\alpha$ & 0.654 & $216.90_{-25.23}^{+25.87}$ & $115.70_{-28.54}^{+29.04}$ \\
\hline Fe XVII & 0.720 & $215.03_{-19.73}^{+20.41}$ & $124.66_{-22.90}^{+23.37}$ \\
\hline Fe XVII & 0.826 & $187.08_{-19.28}^{+19.82}$ & $122.69_{-15.88}^{+16.16}$ \\
\hline Fe XVIII & 0.873 & $\ldots$ & $\ldots$ \\
\hline Ni XIX & 0.884 & $\ldots$ & $\ldots$ \\
\hline $\mathrm{Ne} I X$ & 0.905 & $58.46_{-58.46}^{+90.27}$ & $150.99_{-12.83}^{+13.09}$ \\
\hline Fe XIX & 0.917 & $\ldots$ & $\ldots$ \\
\hline Fe XIX & 0.922 & $\ldots$ & $\ldots$ \\
\hline $\mathrm{Ne} X$ & 1.022 & $95.08_{-7.31}^{+7.51}$ & $57.47_{-8.50}^{+8.70}$ \\
\hline Fe XXIV & 1.129 & $12.30_{-7.06}^{+7.22}$ & $1.64_{-1.64}^{+7.67}$ \\
\hline Fe XXIV & 1.168 & $45.73_{-6.99}^{+7.21}$ & $26.30_{-7.45}^{+6.49}$ \\
\hline Mg XI & 1.331 & $38.72_{-4.00}^{+4.13}$ & $21.53_{-4.33}^{+4.47}$ \\
\hline Mg XI & 1.352 & $\ldots$ & $\ldots$ \\
\hline Mg XII & 1.478 & $12.94_{-2.76}^{+2.89}$ & $3.03_{-2.93}^{+3.05}$ \\
\hline Mg XII & 1.745 & $10.80_{-2.69}^{+2.83}$ & $6.28_{-2.71}^{+2.85}$ \\
\hline Si XIII & 1.839 & $14.26_{-9.24}^{+2.79}$ & $12.31_{-4.44}^{+2.85}$ \\
\hline Si XIII & 1.865 & $<10.81$ & $<4.80$ \\
\hline Si XIV & 2.005 & $6.14_{-2.06}^{+2.21}$ & $4.43_{-2.07}^{+2.23}$ \\
\hline Si XIII & 2.180 & & $\ldots$ \\
\hline S XV & 2.430 & $9.88_{-2.67}^{+2.91}$ & $10.15_{-2.67}^{+2.92}$ \\
\hline $\mathrm{Fe} \mathrm{K} \alpha$ & 6.442 & $29.85_{-7.83}^{+7.99}$ & $33.02_{-7.40}^{+8.14}$ \\
\hline \multicolumn{4}{|c|}{ Basic Parameters } \\
\hline$\Gamma$ & $\ldots$ & $=1.8^{\mathrm{a}}$ & $=3.11_{-0.13}^{+0.13}$ \\
\hline L-statistic & $\ldots$ & 600.26 & 556.67 \\
\hline \multirow[t]{2}{*}{$\chi^{2} /$ dof } & $\ldots$ & $770.18 / 545$ & $640.24 / 544$ \\
\hline & & $=1.41$ & $=1.18$ \\
\hline
\end{tabular}

Note.

${ }^{\mathrm{a}}$ For this fit, the power-law index of the continuum has been fixed to $\Gamma=1.8$.

images show that the brightest regions do not perfectly correlate with regions of high $[\mathrm{O} \mathrm{III]} / \mathrm{H} \alpha$ or $[\mathrm{S} \mathrm{II}] / \mathrm{H} \alpha$. The morphologies of these line ratios occupy complementary spatial regions, implying multiphase distribution of the gas.

We now gain a more detailed picture of the physical processes from the X-ray line emission via adaptive smoothing, spatially resolved line hardness ratios, and multiple binning approaches. Such approaches are necessary even for such a bright Sy2 as NGC 3393, due to the limited number of photons per energy band. The morphology of different X-ray species can then be used to investigate the physical processes behind their excitation.

Inter-species morphological differences are evident to the eye in Figure 2, where Chandra band-specific images, including Ne IX, O VIII, and O VII, are directly compared with radio emission and [O $\mathrm{III}]$. A more rigorous approach in Sections 2.1.3-2.2 demonstrates that these differences are significant when comparing Ne IX and O VII.

1. The peaks of Ne IX emission are associated with the leading edges of the radio outflows.
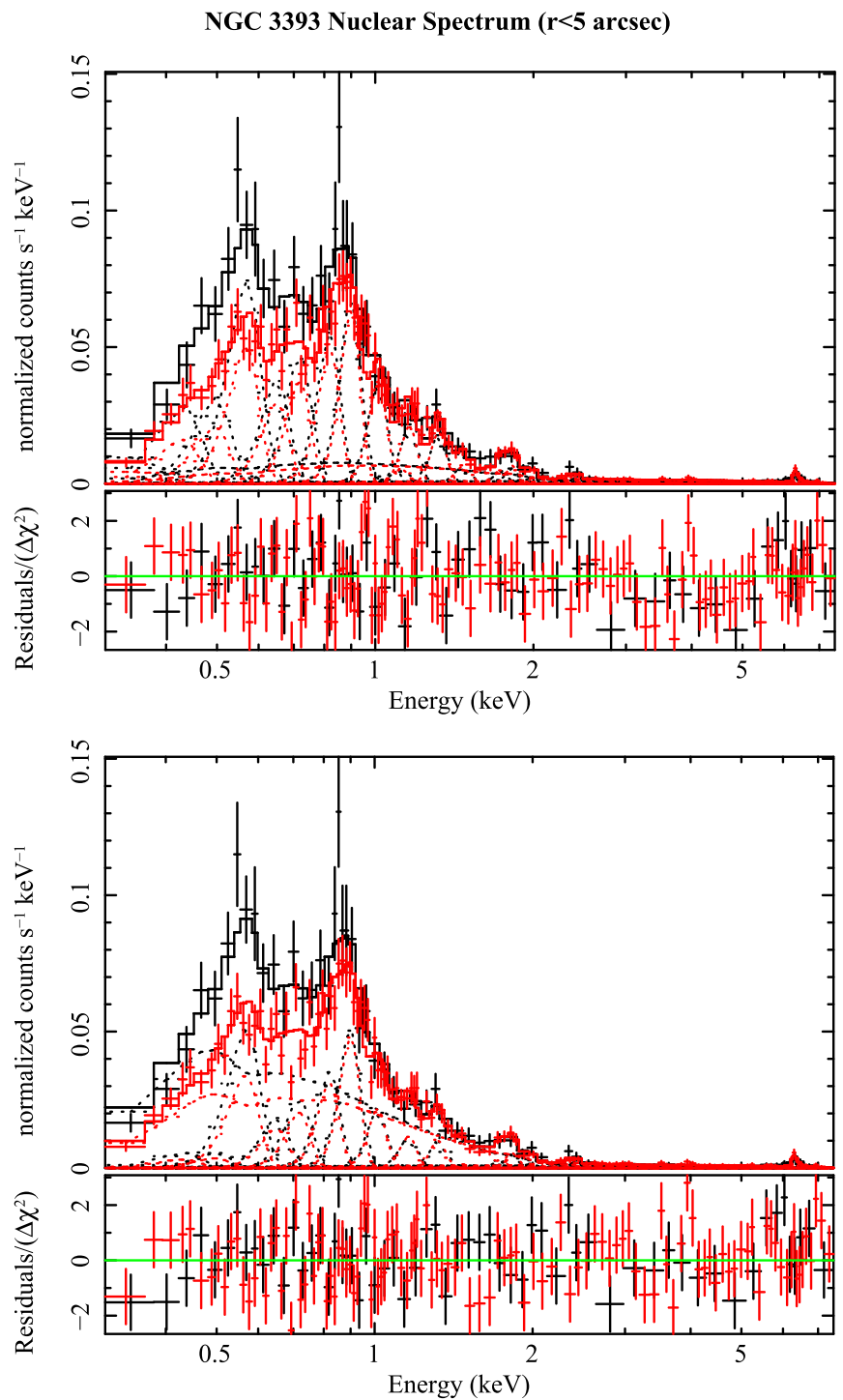

Figure 9. Spectral fits to X-rays detected by Chandra within $r=5^{\prime \prime}$ from the nucleus of NGC 3393. In addition to a spatially unresolved nuclear component (fit separately), each model has a neutral host absorption component. The extended plasma is modeled as a power-law continuum plus multiple discrete emission lines described as Gaussians with fixed transition energies, as described in Table 3. Red and black data points are from obsids 12290 and 4868 , respectively. Dashed lines indicate model components. Residuals from models are depicted in the lower panels. Top: the continuum power-law index is fixed to Gamma $=1.8$. Bottom: the power-law index is fitted as a free component.

2. Strong O VII coincides with strong Ne IX leading the SW outflow (and stronger but less extended radio emission).

3. Strong O VII does not overlap with the Ne IX leading the NE outflow.

4. Radial profiles are less useful for identifying these deviations for X-ray species ratios, which are more significant in e.g., Ne IX-O VII HR maps (Figure 4).

5. Radial profiles show deviations of $[\mathrm{O} \mathrm{III]} /(0.3-2 \mathrm{keV})$ ratios in the nucleus from $[\mathrm{O} \mathrm{III]}] /(0.3-2 \mathrm{keV})$ at large radii.

6. Elevated [O III] is clearly associated with regions at $\lesssim 1^{\prime \prime}$ external to the radio peaks.

7. X-ray emission is elevated both inside these radiodominated regions and immediately outside the 

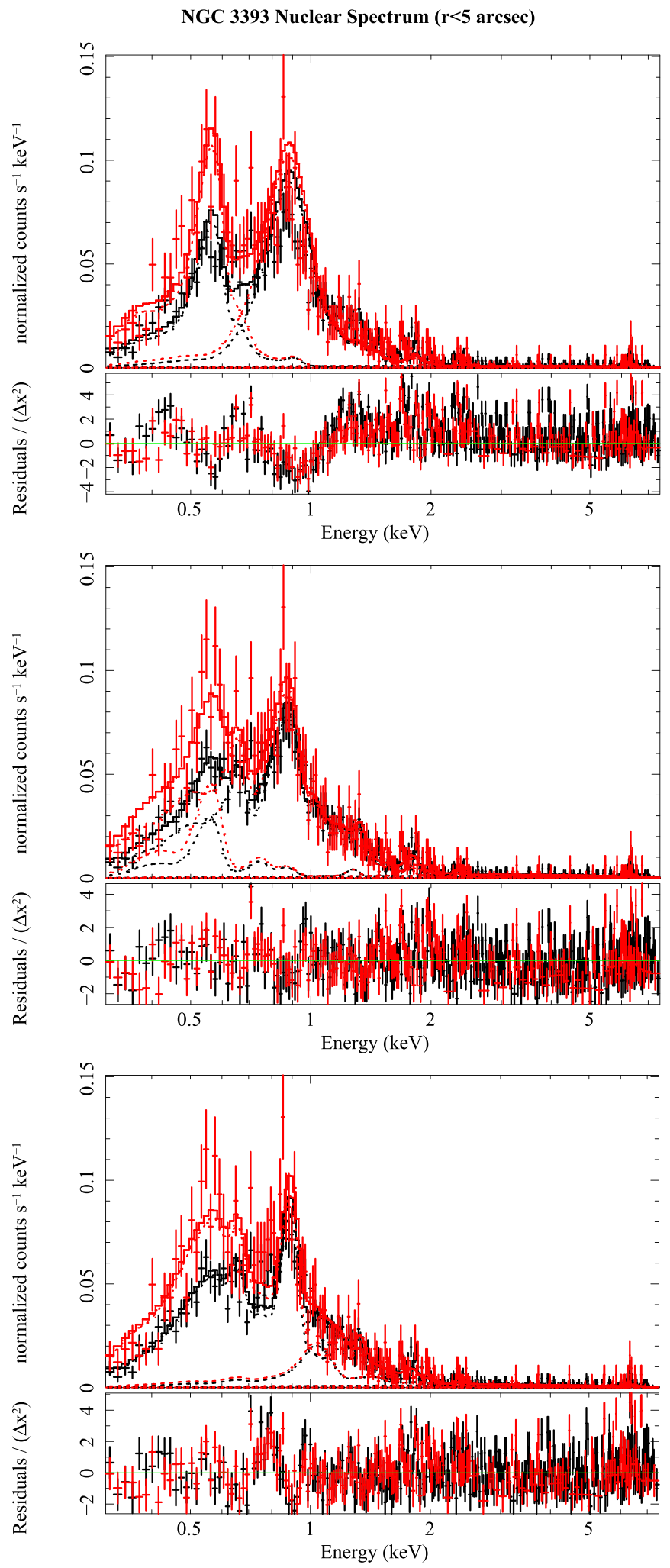

Figure 10. As in Figure 9, but for the different combinations of APEC and CLOUDY plasma model components in Table 4. Top: two APEC thermal plasma components. Middle: two CLOUDY components. Bottom: a combination of single APEC and CLOUDY components. The APEC component peaks near $1 \mathrm{keV}$.

$[\mathrm{O} \mathrm{III}] /(0.3-2 \mathrm{keV})$ peaks. The major exception to this rule is the $\left[\mathrm{O} \mathrm{III]} /(0.3-2 \mathrm{keV})\right.$ peak at $r \sim 5^{\prime \prime} \mathrm{SW}$ of the nucleus.
Table 4

Plasma Model Fits for $r<5^{\prime \prime}$

\begin{tabular}{|c|c|c|}
\hline Component & Parameter & Value \\
\hline \multicolumn{3}{|c|}{ Single APEC and Single CLOUDY } \\
\hline zphabs & $N_{\mathrm{H}}\left(\times 10^{20} \mathrm{~cm}^{-2}\right)$ & a \\
\hline apec & $\log F_{X}^{\mathrm{b}}\left(\operatorname{erg~cm}{ }^{-2} \mathrm{~s}^{-1}\right)$ & $-13.27_{-0.06}^{+0.09}$ \\
\hline & $k T(\mathrm{keV})$ & $1.50_{-0.11}^{+0.18}$ \\
\hline \multirow[t]{3}{*}{ cloudy } & $\log F_{X}^{\mathrm{b}}\left(\operatorname{erg~cm}{ }^{-2} \mathrm{~s}^{-1}\right)$ & $-12.42_{-0.02}^{+0.01}$ \\
\hline & $\log U$ & $0.55_{-0.03}^{+0.03}$ \\
\hline & $\log N_{\mathrm{H}}\left(\mathrm{cm}^{-2}\right)$ & $21.32_{-0.15}^{+0.17}$ \\
\hline \multirow[t]{2}{*}{ Fit } & L-statistic & 702.82 \\
\hline & $\chi^{2} / \mathrm{dof}^{\mathrm{b}}$ & $780.43 / 566=1.38$ \\
\hline \multicolumn{3}{|c|}{ Double APEC } \\
\hline zphabs & $N_{\mathrm{H}}\left(\times 10^{20} \mathrm{~cm}^{-2}\right)$ & a \\
\hline apec & $\log F_{X}^{\mathrm{b}}\left(\mathrm{erg} \mathrm{cm}^{-2} \mathrm{~s}^{-1}\right)$ & $-12.64_{-0.02}^{+0.03}$ \\
\hline \multirow{3}{*}{ apec } & $k T(\mathrm{keV})$ & $0.13_{-0.02}^{+0.03}$ \\
\hline & $\log F_{X}^{\mathrm{b}}\left(\mathrm{erg} \mathrm{cm}^{-2} \mathrm{~s}^{-1}\right)$ & $-12.77_{-0.01}^{+0.03}$ \\
\hline & $k T(\mathrm{keV})$ & $0.84_{-0.11}^{+0.01}$ \\
\hline \multirow[t]{2}{*}{ Fit } & L-statistic & 1065.92 \\
\hline & $\chi^{2} /$ dof $^{b}$ & $821.03 / 567=1.45$ \\
\hline \multicolumn{3}{|c|}{ Double CLOUDY } \\
\hline zphabs & $N_{\mathrm{H}}\left(\times 10^{20} \mathrm{~cm}^{-2}\right)$ & 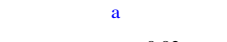 \\
\hline cloudy & $\log F_{X}^{\mathrm{b}}\left(\mathrm{erg} \mathrm{cm}^{-2} \mathrm{~s}^{-1}\right)$ & $-12.79_{-1.01}^{+0.92}$ \\
\hline \multirow{5}{*}{ cloudy } & $\log U$ & $-0.53_{-0.02}^{+0.03}$ \\
\hline & $\log N_{\mathrm{H}}\left(\mathrm{cm}^{-2}\right)$ & $23.5_{-2.00}^{+0.71}$ \\
\hline & $\log F_{X}^{\mathrm{b}}\left(\mathrm{erg} \mathrm{cm}^{-2} \mathrm{~s}^{-1}\right)$ & $-12.49_{-0.76}^{+0.69}$ \\
\hline & $\log U$ & $0.80_{-0.05}^{+0.06}$ \\
\hline & $\log N_{\mathrm{H}}\left(\mathrm{cm}^{-2}\right)$ & $20.16_{-0.92}^{+1.69}$ \\
\hline \multirow[t]{2}{*}{ Fit } & L-statistic & 653.95 \\
\hline & $\chi^{2} /$ dof $^{b}$ & $927.42 / 574=1.64$ \\
\hline
\end{tabular}

Notes.

${ }^{\text {a }}$ Negligible $\left(N_{\mathrm{H}}<10^{17}\right)$.

b $0.3-8.0 \mathrm{keV}$.

${ }^{\mathrm{c}}$ Measured for the best lstat under Xspec.

Noting these trends, we examine the spectral fits described in Section 2.2, which are shown in Tables 3-5 and Figures 9-11.

1. Gaussian modeling fails to measure Fe XIX and other lines around Ne IX, which are resolved and detected by Koss et al. (2015) using grating spectroscopy.

2. Instead, these models of lower-resolution CCD spectra prefer Ne IX, which is inferred to be weak in Koss et al. (2015).

3. Several other CCD-derived lines in Table 3 directly contradict grating measurements by Koss et al. (2015) where the separation is too small for CCD energy resolution

4. The simplest models of the ENLR emission suggest domination by photoionizing radiation from the AGN with a possible contribution from a thermal component.

5. The nucleus (region 9, containing the unresolved AGN) is well described by photoionizing radiation from the AGN through a highly obscuring $\left(\log N_{\mathrm{H}} \sim 23.5 \mathrm{~cm}^{-2}\right)$ medium. 
Table 5

Region Model Fits

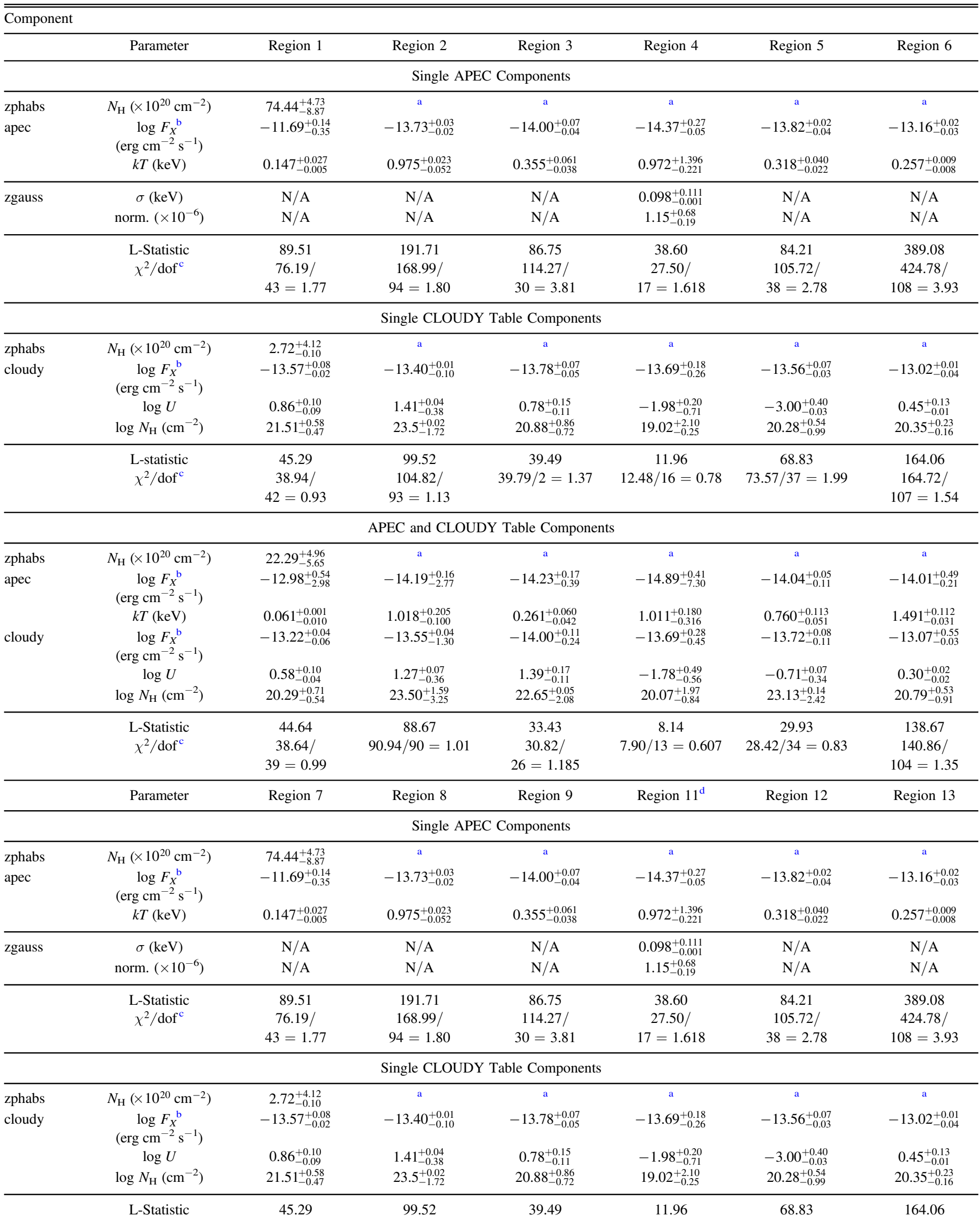


Table 5

(Continued)

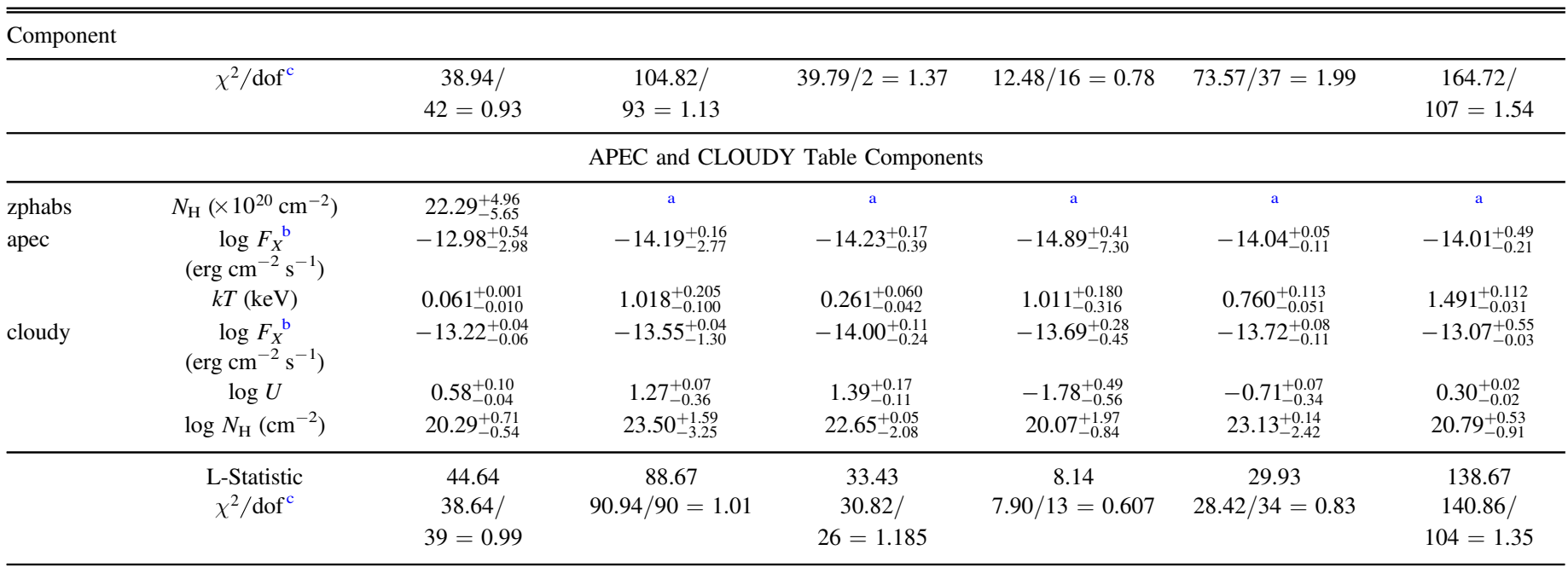

Notes. Region 10 contains the larger part of several bright spatially resolved regions $(4,5,9,10$, and 11) and is therefore excluded.

a negligible $\left(N_{\mathrm{H}}<10^{17}\right)$.

b $0.3-8.0 \mathrm{keV}$.

${ }^{c}$ measured for the best lstat under Xspec.

${ }^{\mathrm{d}}$ Region 11 has an excess of $\mathrm{Fe} \mathrm{K} \alpha$, which is modeled in all fits by a single Gaussian at $6.4 \mathrm{keV}$ (host frame).

6. Elevated nuclear emission associated with the Ne IX complex may be due to a collisional plasma, but the flux of this component has a large lower bound.

7. The E-W O VII cross bar (regions 4 and 5) is also well described by photoionizing radiation from the AGN through a highly obscuring $\left(\log N_{\mathrm{H}} \sim 23.5 \mathrm{~cm}^{-2}\right)$ medium.

8. In general, spatially resolved spectroscopy of individual regions suggests lower $k T$ values than obtained from the best-fit value of the entire nucleus within $r=5^{\prime \prime}$.

9. AGN photoionization is most clearly dominant over emission from a collisional plasma in at least two regions in the bicone which are external to the radio emission (2 and 13$)$.

10. CLOUDY + APEC models of the cross-cone regions (3 and 7) is consistent with AGN photoionization through a lower-density $\left(\log N_{\mathrm{H}} \sim 20.2 \mathrm{~cm}^{-2}\right) \quad$ medium. The strength of any thermal plasma is correlated with the assumed obscuration.

11. Emission in two regions that have elevated Ne IX and are associated with radio outflows $(9,12)$ is consistent with having a collisional component with flux comparable to reprocessed photoionizing emission.

12. Region 11 in the near-SE bicone has some atypical properties for these regions. It is bright in the Ne IX complex but not associated with radio emission, and appears to be photoionization-dominated (with an atypically low ionization parameter for this region). Its models require an additional $\mathrm{Fe} \mathrm{K} \alpha$ component beyond simple AGN scaling, and at $r \sim 1^{\prime \prime}$ from the AGN, contamination by the AGN point source should be low.

13. Some regions in the bicone (e.g., 1, 2, and 6) permit a significant collisional component even though they are photoionization dominated.

\section{Discussion}

\subsection{Emission Line Morphology}

\subsection{1. [O III] and the AGN Wind}

Wang et al. (2011a) investigated the morphology of X-ray emission from the ENLR of NGC 4151 and found spectroscopic evidence for shock excitation on the leading edges of the sub-kpc jets, which coincides with arcs of enhanced $\mathrm{O}$ III emission that wrap around the jet lobes. Paggi et al. (2012) likewise found spectroscopic evidence for shock excitation associated with the arcs of $\mathrm{O}$ III emission that lead the jet lobes of Mrk 573. If the multi-wavelength evidence for shock excitation noted in Papers I and II has its origin in photoionizing shocks leading the bipolar outflows, then spatially resolved spectroscopy of the ENLR should show similar evidence.

The [O III] $/ 0.3-2 \mathrm{keV}$ ratio is sensitive to density, and if an AGN wind has a uniform spectrum of densities at a given distance, then simple assumptions of an $r^{-2}$ density profile imply that this ratio should be flat with increasing radius, since the ionization parameter then will not change (Bianchi et al. 2006; Wang et al. 2011a). But increased [O III]/0.3-2 keV has been observed in association with possible shock locations by Paggi et al. (2012). We see similar trends here, such that areas of excess [O III]/0.3-2 keV are associated with the leading edges of radio outflows. The [O III] $/ 0.3-2 \mathrm{keV}$ normalization is comparable to values found in previous studies of Mrk 573 and NGC 4151 (Wang et al. 2011a; Paggi et al. 2012) when their values are corrected by converting HST rms bandwidth to FWHM bandwidth. ${ }^{13}$

The $[\mathrm{O}$ III] $/ 0.3-2 \mathrm{keV}$ spikes that we observe could be consistent with local overdensities, such as clearly associated

\footnotetext{
13 Rms bandwidth appears to have been used in Paggi et al. (2012) and Wang et al. (2011a), which gives an incorrect low value for [O III] $/(0.3-2 \mathrm{keV})$. We have confirmed this by directly examining their archival data.
} 


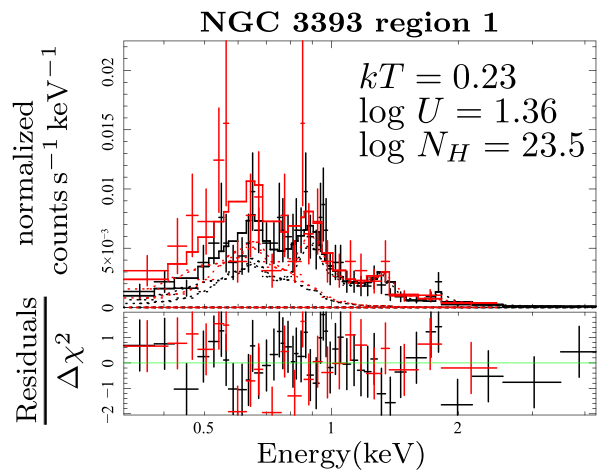

NGC 3393 region 4

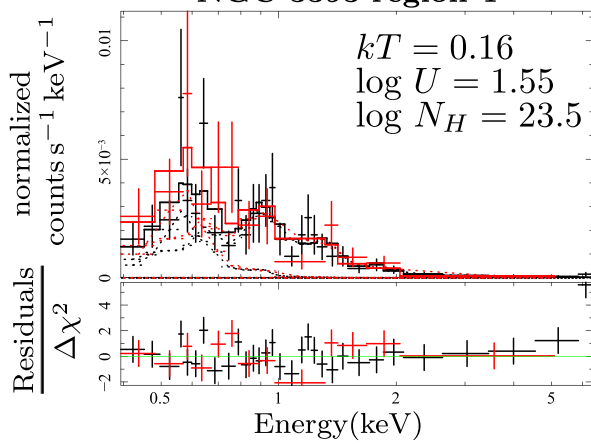

NGC 3393 region 7
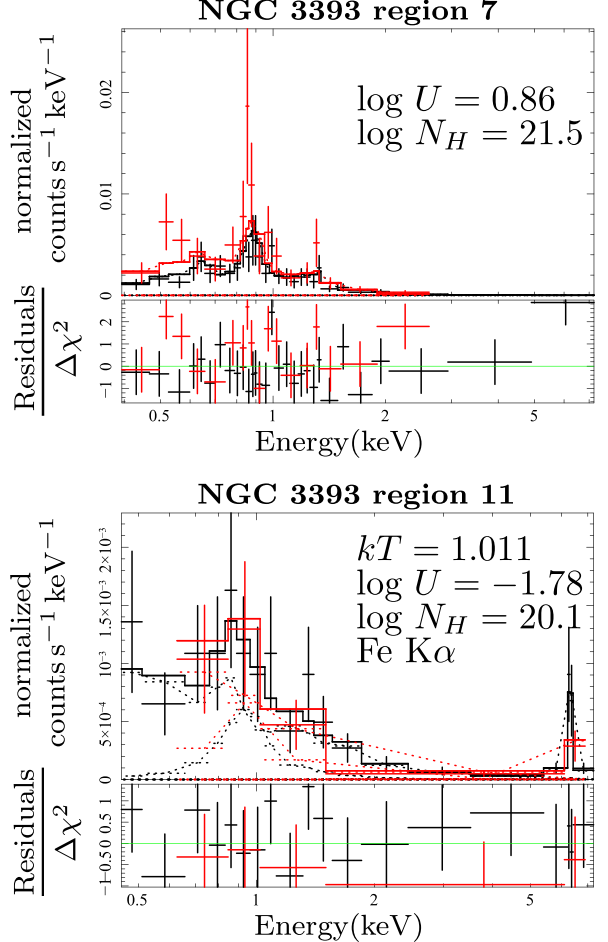

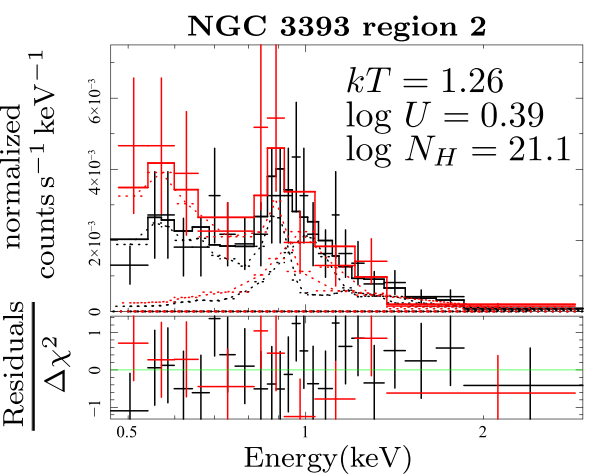

Energy $(\mathrm{keV})$
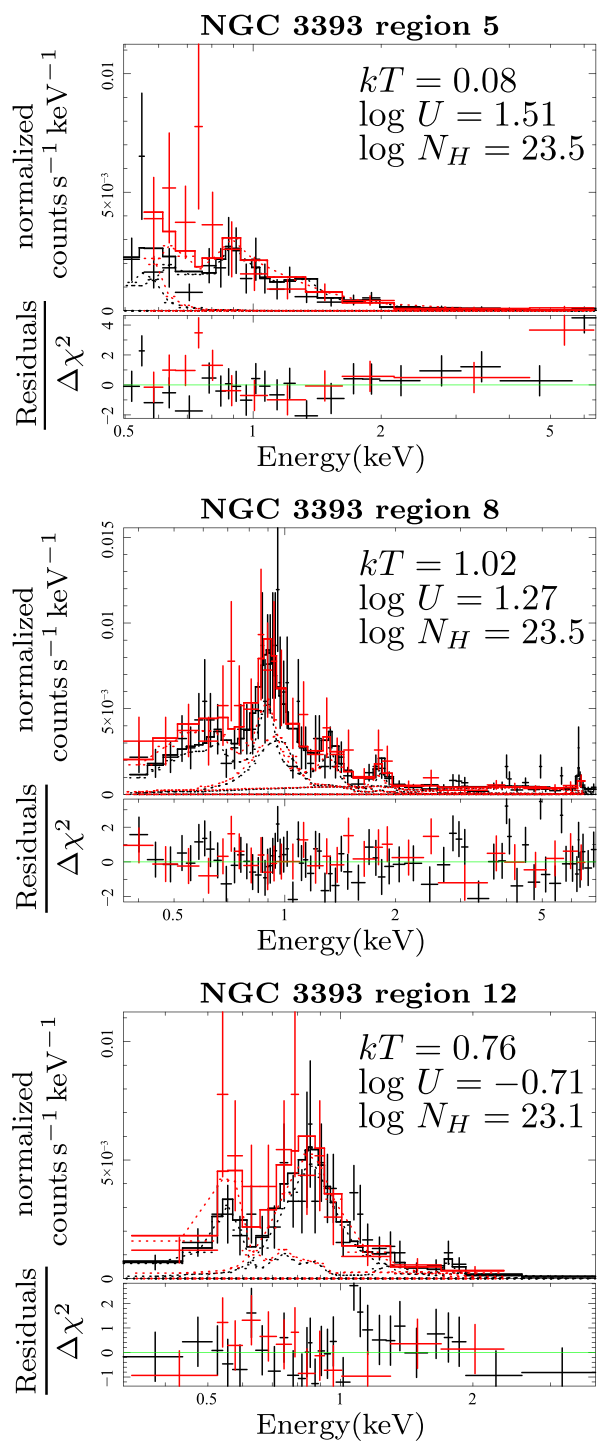

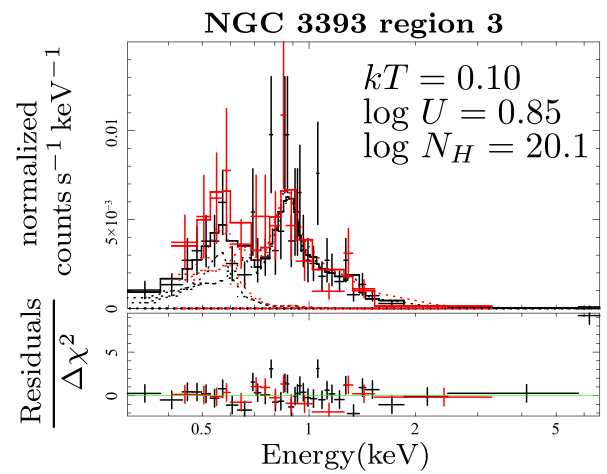

NGC 3393 region 6

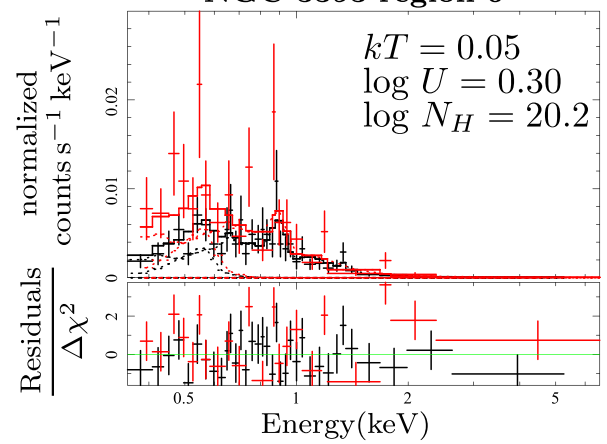

NGC 3393 region 9
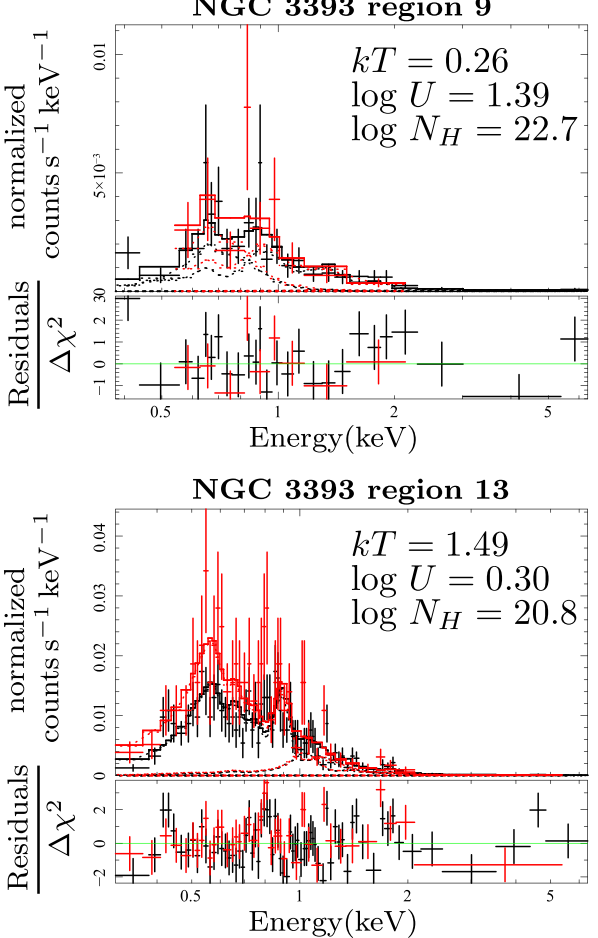

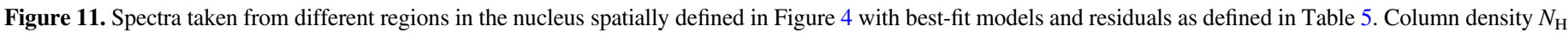
refers to the reprocessing CLOUDY component, not neutral absorption. Plots are otherwise similar to Figure 9.

with a spiral arms intersecting the bicone (at $r \sim 5^{\prime \prime} \mathrm{SW}$ ), and possibly associated with shocks or ISM compression at $r \sim 1$ " SW and NE. A "local overdensity" interpretation would be consistent with the spike at $r \sim 5^{\prime \prime} \mathrm{SW}$, where there is no obvious optical or radio evidence for outflow-ISM interaction. Rather, the bicone intersects with a spiral dust lane at that point.

\subsubsection{X-Ray Line Enhancements as Physical Tracers}

The presence of enhanced emission in the Ne IX band is consistent with other findings in Mrk 573 (Paggi et al. 2012) and NGC 4151 (Wang et al. 2011a). In both cases, although the bulk of the X-ray emission for the ENLR is associated with reflection of an incident AGN spectrum by a photoionized medium, regions immediately adjacent to outflow-ISM 
interactions (indicated by radio emission and bright optical/ $\mathrm{X}$-ray emission) demonstrate spectra that are better represented by emission from a thermal plasma, as might be expected from shock interactions or from overdensities in a hot X-ray emitting outflow. As noted in Paper II, although the optical line ratios within the bicone are consistent with photoionization, shocks are not excluded since kinematics from the HST Space Telescope Imaging Spectrograph (STIS) in Fischer et al. (2013) are consistent with shocks fast enough to photoionize the precursor ISM.

An E-W O VII cross-bar is evident at subarcsecond scales. It neatly traces a similar structure in [O III], but is stronger at $r<1^{\prime \prime}$ (versus $r>1^{\prime \prime}$ for [O III]). This appears to correspond to the nuclear bar (described in greater detail by AlonsoHerrero et al. 1998; Läsker et al. 2016; Finlez et al. 2018). The $\mathrm{O}$ VII bar therefore likely corresponds to highly ionized gas associated with the black hole's fueling flows, but could trace material of a different density or ionization state than gas in the bar at $r>1^{\prime \prime}$.

\subsubsection{CCD Imaging Spectroscopy and Grating Measurements}

Unlike CCD spectroscopy (FWHM $\Delta E \sim 100 \mathrm{eV}$ ), grating spectroscopy with Chandra can resolve closely spaced diagnostic lines. We therefore prefer measurements of individual emission lines by Koss et al. (2015) in assessing the overall state of the NGC 3393 plasma when those measurements directly contradict our Gaussian models of the CCD spectrum (as integrated over the entire nucleus, shown in Table 3).

The Nature of the Ne IX Complex-In Section 2.2, we show that X-ray spectroscopy suggests that an apparent excess in $\mathrm{X}$-ray emission in the ENLR near Ne IX could be explained by a simple two-component model where the bulk of emission is photoionized, since a single-component photoionization model is unable to account for this excess.

Although we continue to designate this spectral excess as "Ne IX" due to its proximity relative to the triplet at $E=0.915$ $\mathrm{keV}$ (rest), in practice this narrow band refers to a complex where the low energy resolution of ACIS-S blends emission from multiple lines, including Fe XVIII, Fe XIX, and Ni XIX. The complexity of this region is evident in grating spectroscopy from Koss et al. (2015). They attribute relatively little emission to Ne IX, but two factors complicate this interpretation. First, their spectrum is spatially unresolved and emission from a collisional plasma (for example) could be diluted by the bulk of the emission, which is due to photoionization. Second, the $0.906 \mathrm{keV} 1 \mathrm{~s}^{2}{ }^{2} \mathrm{~S}_{0}-1 \mathrm{~s} 2 \mathrm{~s}^{3} \mathrm{~S}_{1}$ Ne IX transition is the forbidden component of a triplet where the two higher-energy lines are difficult to distinguish from Fe XIX even with the grating. As a result of the expected ratios within the Ne IX triplet and the ratio of the two Fe XIX blends, we can expect about half of the emission from the Ne IX complex to arise from Fe XIX in a collisional plasma. Relatively little Fe XIX is expected from a photoionization-dominated plasma, in which case Ne IX would dominate this band.

The Ne IX associated with shocks in the NGC 3393 outflows could originate from a shock-photoionized precursor, rather than from collisional plasma in the shocks themselves (S. Kraemer 2018, personal communication). But temperatures of peak ionization fractions for Ne IX and Fe XIX (at $k T \sim 0.1$ and $\sim 0.7 \mathrm{keV}$, respectively) are consistent with our best-fit models.
$\mathrm{O}$ VIII is also roughly cospatial to Ne IX, which is expected given those species experience similar excitation trends with $k T$ and $U$.

Emission Line Ratios from a Collisional Plasma-Other line measurements by Koss et al. (2015) also point to collisional excitation. The ratio of the Fe XVII lines at 0.720 and $0.826 \mathrm{keV}$ is a little above 1 in a collisional plasma (comparable to those observed by Koss et al. 2015), but far above 1 in a photoionized one (Liedahl et al. 1990). The Koss et al. (2015) Fe XVII line ratios indicate a collisionally excited plasma. The helium-like triplets of Mg XI and Si XIII are even more clarifying than Ne IX: the middle intercombination lines are unresolved, the longer forbidden line is weak, and the shorter resonance line is strong, all of which point to a collisional plasma (Porquet \& Dubau 2000). For He-like ions, the forbidden ${ }^{3} \mathrm{~S}_{1}$-ground triplet line is weaker than the resonance ground- ${ }^{1} \mathrm{P}_{1}$ line in collisional equilibrium, but it is stronger in a photoionized plasma due to recombination (Bautista \& Kallman 2000).

Due to the complexity of the system and the hard X-ray luminosity of the AGN, we do expect photoionized excitation to be present alongside collisional excitation in the bicone. The observed $\mathrm{Fe} \mathrm{K} \alpha$ in NGC 3393, for example, should be produced by photoionization of gas with a low ionization state. But the line ratios here are clearly inverted from the case of AGN known to be dominated by photoionization, such as NGC 1068 (as shown by Kinkhabwala et al. 2002, using XMMNewton spectroscopy). NGC 1068 (unlike NGC 3393) also shows multiple strong radiative recombination continuum (RRC) lines expected of photoionization, whereas RRC lines are weak or absent in NGC 3393 (The O VIII RRC may overlap with the Fe XVIII $0.873 \mathrm{keV}$ line claimed by Koss et al. 2015, but in the case of a photoionized plasma the O VIII RRC should be twice as bright as the observed Fe XVIII).

We therefore infer that X-ray grating spectroscopy requires a strong collisional component. Our APEC models derived from spatially resolved CCD spectroscopy are therefore necessary tools to describe kinetic feedback in NGC 3393, and should therefore be investigated in the context of outflows observed at other wavelengths and shock generation.

\subsection{X-Ray Spectroscopy Connections to Optical/ Millimeter Data}

Spatially resolved spectroscopy (as described in Section 2.2) may be key to interpreting the X-ray ENLR near the nucleus of AGN, since the X-rays imply a complex picture. Almost all ENLR regions that we define and investigate are consistent with a model that combines photoionization with a collisional plasma, but the properties of those models vary across the ENLR. Photoionization tends to dominate emission in all regions, but the flux contribution from collisional plasma can approach comparable levels, including throughout the S-shaped arms where the O VII-bright nuclear bar (forming part of the S-shaped arm) appears dominated by highly ionizing radiation reprocessed by nearly Compton-thick material.

\subsubsection{Hot Collisional Gas and Optical Outflows}

"Ne IX-bright" material leading the radio lobes (regions 9 and 12) can be addressed in the context of Finlez et al. (2018), who recently used Gemini Multi-Object Spectrograph Integrated Field Unit (GMOS IFU) data to identify regions of NE 
Table 6

Local Physical Parameters of Collisionally Ionized Gas Derived from Best-fit APEC Models

\begin{tabular}{|c|c|c|c|c|c|c|c|c|}
\hline $\begin{array}{l}\text { Region } \\
\text { (Figure 4) }\end{array}$ & $\begin{array}{c}n_{e} \\
\left(\mathrm{~cm}^{-3}\right)\end{array}$ & $\left(10^{-10} \begin{array}{c}p_{\text {th }} \\
\left.\text { dyne } \mathrm{cm}^{-3}\right)\end{array}\right.$ & $\begin{array}{c}E_{\mathrm{th}} \\
\left(10^{53} \mathrm{erg}\right)\end{array}$ & $\begin{array}{c}t_{\mathrm{cool}} \\
\left(10^{6} \mathrm{yr}\right)\end{array}$ & $\begin{array}{c}c_{s} \\
\left(\mathrm{~km} \mathrm{~s}^{-1}\right)\end{array}$ & $\begin{array}{c}v_{\mathrm{sh}} \\
\left(\mathrm{km} \mathrm{s}^{-1}\right)\end{array}$ & $\begin{array}{c}t_{\text {cross }} \\
\left(10^{5} \mathrm{yr}\right)\end{array}$ & $\begin{array}{c}E_{\text {th }} / t_{\text {cross }} \\
\left(10^{41} \mathrm{erg} \mathrm{s}^{-1}\right)\end{array}$ \\
\hline 1 & $0.33_{-0.04}^{+0.06}$ & $2.46_{-0.25}^{+0.22}$ & $14.86_{-1.65}^{+1.49}$ & $11.06_{-11.06}^{+4.38}$ & $245_{-37}^{+34}$ & $421+59$ & $5.51_{-0.95}^{+1.00}$ & $0.86_{-0.18}^{+0.17}$ \\
\hline 2 & $0.48_{-0.16}^{+0.18}$ & $19.52+9.22$ & $20.61+9.98$ & $41.29{ }_{-11.66}^{+26.23}$ & $5744_{-102}^{+195}$ & $984_{-175}^{+334}$ & $1.044_{-0.37}^{+0.21}$ & $6.27{ }_{-1.78}^{+3.76}$ \\
\hline 3 & $0.33_{-0.06}^{+0.02}$ & $1.05_{-0.17}^{+0.19}$ & $54.77{ }_{-8.84}^{+9.93}$ & $21.39_{-12.98}^{+20.97}$ & $162+34$ & $277+59$ & $21.19_{-4.97}^{+4.74}$ & $0.82_{-0.23}^{+0.24}$ \\
\hline 4 & $0.77_{-0.09}^{+0.09}$ & $3.94{ }_{-0.43}^{+0.51}$ & $4.70_{-0.70}^{+0.77}$ & $5.17_{-4.75}^{+1.51}$ & $205_{-21}^{+29}$ & $351_{-37}^{+50}$ & $3.48_{-0.61}^{+0.50}$ & $0.43_{-0.09}^{+0.10}$ \\
\hline 5 & $3.34{ }_{-0.89}^{+0.90}$ & $8.54_{-1.38}^{+3.77}$ & $4.13_{-0.93}^{+1.94}$ & $3.22+2.59$ & $145_{-14}^{+46}$ & $248_{-24}^{+80}$ & $3.55_{-1.19}^{+0.49}$ & $0.37_{-0.10}^{+0.21}$ \\
\hline 6 & $5.46_{-2.04}^{+6.17}$ & $8.74_{-3.15}^{+0.37}$ & $636.06_{-229.27}^{+27.94}$ & $11.35_{-7.12}^{+10.12}$ & $114_{-34}^{+11}$ & $1966_{-59}^{+20}$ & $21.54_{-3.05}^{+6.81}$ & $9.38_{-4.50}^{+1.39}$ \\
\hline 7 & $1.56_{-0.74}^{+5.30}$ & $3.05_{-0.50}^{+0.07}$ & $156.05_{-25.80}^{+4.24}$ & $14.29{ }_{-14.29}^{+14.28}$ & $126_{-26}^{+8}$ & $217{ }_{-44}^{+14}$ & $29.48{ }_{-3.50}^{+6.66}$ & $1.68_{-0.47}^{+0.20}$ \\
\hline 8 & $0.70_{-0.21}^{+0.11}$ & $22.90_{-4.10}^{+5.74}$ & $12.27_{-2.86}^{+3.58}$ & $18.22{ }_{-9.16}^{+18.95}$ & $517{ }_{-81}^{+116}$ & $886_{-139}^{+199}$ & $1.14_{-0.28}^{+0.21}$ & $3.43_{-1.02}^{+1.31}$ \\
\hline 9 & $0.75_{-0.14}^{+0.19}$ & $6.24_{-1.37}^{+1.72}$ & $3.35_{-0.89}^{+1.05}$ & $5.45_{-2.99}^{+3.65}$ & $261_{-52}^{+63}$ & $447{ }_{-90}^{+107}$ & $2.25_{-0.59}^{+0.51}$ & $0.47_{-0.16}^{+0.19}$ \\
\hline 11 & $0.36_{-0.06}^{+0.06}$ & $11.73_{-4.06}^{+2.73}$ & $6.29_{-2.37}^{+1.74}$ & $46.78_{-46.78}^{+48.54}$ & $515_{-144}^{+109}$ & $882{ }_{-247}^{+186}$ & $1.14_{-0.27}^{+0.34}$ & $1.75_{-0.84}^{+0.63}$ \\
\hline 12 & $0.64_{-0.10}^{+0.12}$ & $15.56_{-2.21}^{+3.03}$ & $11.89_{-2.25}^{+2.75}$ & $12.49_{-2.82}^{+4.02}$ & $446_{-58}^{+86}$ & $765_{-99}^{+147}$ & $1.48_{-0.32}^{+0.24}$ & $2.54{ }_{-0.64}^{+0.81}$ \\
\hline 13 & $0.23{ }_{-0.07}^{+0.08}$ & $10.94{ }_{-0.42}^{+0.90}$ & $122.95{ }_{-6.23}^{+10.85}$ & $120.62{ }_{-120.62}^{+47.45}$ & $625_{-45}^{+86}$ & $1071_{-77}^{+147}$ & $5.022_{-0.85}^{+0.62}$ & $7.77{ }_{-1.04}^{+1.49}$ \\
\hline
\end{tabular}

overdensity, NE outflow, SW radial outflow, and SW equatorial outflow, which they label "O1," "O2," "O3," and "O4," respectively (Figures 6 and 7). In the shock interpretation for the NE cone advanced by Finlez et al. (2018), we would expect a hot collisional component associated with outflowing $\mathrm{O} 1$ and higher-density $\mathrm{O} 2$, and we find this with region 12 , which is located between them. In our region 12, the collisional component is comparable to the reprocessed component and is relatively hot $(k T \sim 0.76 \mathrm{keV})$, as might be expected in a shock scenario.

In the SW cone, region 9 is clearly associated with outflow $\mathrm{O} 3$ and has a significant collisional component, but unlike region 12 the "Ne IX" excess is attributable to the best-fit photoionization component rather than collisional plasma, since the best-fit collisional temperature is lower than in region $12(k T \sim 0.26 \mathrm{keV})$. The reason for this is not clear. Region 9 is closer to the nucleus and may thus encounters more direct photoionization ( $\log U \sim 1.39$ versus $\sim-0.71$ ), or the lateral outflow may prevent pileup of the outflowing plasma near O3. Alternately, the detected number of photons may be insufficient to distinguish the best-fit model in region 9 from a true solution that is closer to region 12 .

Region 11 is spatially consistent with the equatorial outflow O4 identified by Finlez et al. (2018). The origin of the Fe $\mathrm{K} \alpha$ component here is uncertain. Region 11 is close enough to the off-center Fe $\mathrm{K} \alpha$ source (proposed by Fabbiano et al. 2011, as a secondary $\mathrm{SMBH}$ ) that it could be contaminated by photons from that source. We do not find evidence for obscuration, which might be expected from the disk if the outflow were behind it. The collisional component appears hot $(k T \sim 1$ $\mathrm{keV}$ ) but weak, $F_{X} \lesssim$ an order of magnitude below the reprocessed photoionization.

\subsubsection{AGN Outflows and the Cross-Cone}

The cross-cone (regions 3 and 7) is consistent with reprocessing by a smaller column $\left(\log N_{\mathrm{H}} \sim 20.2 \mathrm{~cm}^{-2}\right.$ ), as might be expected from a leaky torus. These regions permit significant cold absorption $\left(N_{\mathrm{H}} \gtrsim\right.$ few $\left.\times 10^{20} \mathrm{~cm}^{-2}\right)$, which is unsurprising given the cospatial Atacama Large Millimeter Array (ALMA) detection of CO by Finlez et al. (2018), e.g., Figure 9. A cooler $(k T \lesssim 0.1 \mathrm{keV})$ collisional component is permitted and could be associated with the tail end of an equatorial outflow, but has very uncertain $F_{X}$ that is correlated with column density due to the low temperature (and indeed the single CLOUDY model with no APEC is preferred in region 7).

Wang et al. (2011b) describe a similar scenario based on observations of elevated obscuration in the cross-cone of NGC 4151, which is associated with strong $\mathrm{CO}$ emission. A cocoon of collisionally excited gas may therefore be common in the presence of AGN feedback (as expected by, e.g., Mukherjee et al. 2016 when outflows are confined by the local ISM). Equatorial outflows are commonly seen in optical and infrared IFU data, such as in NGC 5929 (Riffel et al. 2015), NGC 1386 (Lena et al. 2015, and 3C 33 (Couto et al. 2017), and may therefore produce similar X-ray signatures.

\subsection{Constraints on Feedback from X-Ray Spectroscopy}

We examine the feedback effects of the AGN on the surrounding ISM in a similar manner to that of Paggi et al. (2012) and Sartori et al. (2016). The results of these calculations are displayed in Table 6. The thermal energy injected into a given region can be described as $E_{\text {th }}=[\gamma /(\gamma-1)] p_{\text {th }} V$, where $\gamma$ is the heat capacity ratio in the gas $(\gamma=4 / 3$ for a relativistic gas, but here we assume $\gamma=5 / 3$ for a non-relativistic monoatomic gas), $p_{\text {th }}$ is the thermal pressure of the collisional gas, and $V$ is the volume of the region. For circular regions, we assume a sphere. For ellipsoids and rectangular solids, we assume a $z$-axis equal to the smaller dimension in the plane of the sky. For region 13 in the NE cone, we assume a solid "wedge" of thickness 200 pc.

\subsubsection{Pressure and Density}

The emission measure $(E M)$ of the collisional gas is directly measured by the normalization of the APEC model assuming angular distance $D_{A}$. Since

$$
E M=\frac{10^{-14}}{4 \pi D_{A}^{2}(1+z)^{2}} \int n_{e} n_{\mathrm{H}} d V
$$

we can derive $p_{\text {th }}$ from the best-fit $E M$ and $k T$ from the APEC component by assuming an ideal proton-electron gas where $p=2 n_{e} k T$. Typical $n_{e}$ for the X-ray emitting collisional plasma is $\sim 1 \mathrm{~cm}^{-3}$, with $p_{\text {th }} \sim$ few $\times 10^{-10} \mathrm{dyne}^{-3}$. In general, $p_{\text {th }}$ is a factor of $\sim$ few lower than for Mrk 573 (Paggi et al. 2012). The lowest-pressure regions include the cross-cones 
and may be associated with high-velocity material escaping "nozzle" (Finlez et al. 2018), whereas the highest-pressure regions are at the "elbows" of the S-shaped arms, leading the radio blobs. In general the $\mathrm{X}$-ray pressures comparable to those inferred from optical lines such as [O III] $\lambda \lambda 4363,4959,5007$ and [S II] $\lambda \lambda 6716,6731\left(\sim 10^{-9}\right.$ dyne $\mathrm{cm}^{-3}$; Cooke et al. 2000), suggesting the optical and X-ray gas may be coincident and in equilibrium.

Cooke et al. (2000) show that the equipartition pressures in the NE and SW lobes are $2.7 \times 10^{-8}$ dyne $\mathrm{cm}^{-3}$ and $4.3 \times$ $10^{-7}$ dyne $\mathrm{cm}^{-3}$, respectively. This is much higher than for the thermal X-ray plasma, and implies that the jet pressure is easily sufficient to sustain shock heating of the hot gas, and suggests the lobes may be in the process of burrowing outwards.

The large pressure difference between radio and X-ray/ optical deserves some consideration. If [O III] arises from a photoionization precursor with $n \sim 500 \mathrm{~cm}^{-3}$ (based on the pressure), then we expect a density of 2000 behind the shock, as opposed to $n_{e} \sim 1 \mathrm{~cm}^{-3}$ (shown in Table 6). A selfconsistent interpretation might be that the radio lobes with $p \sim$ $10^{-7}$ dyne $\mathrm{cm}^{-3}$ drive shocks into a pre-shock gas with $v \sim 100 \mathrm{~km} \mathrm{~s}^{-1}$. The pre-shock gas would not itself produce $\mathrm{X}$-rays. If some of the gas has $n \sim 10 \mathrm{~cm}^{-3}$, then the shock speed would be $\sim 700 \mathrm{~km} \mathrm{~s}^{-1}$, giving the inferred X-ray temperatures. A filling factor of $\sim 0.1$ would be necessary in the X-ray emitting regions, which could be a thin layer between the radio lobe and optical region. Another possibility would be that most of the volume is filled with much lower density (and much hotter) gas that has such a low EM that it is not seen. These scenarios could affect subsequent estimates of the shock crossing time $t_{\text {cross }}$ and the cooling time $t_{\text {cool }}$.

Since the X-rays and [O III] are cospatial, we might alternatively suppose that the X-ray gas cools to $10,000 \mathrm{~K}$, below which photoionization dominates the energetics. In that case, the pressure in the [O III] gas would be equal to that of the $\mathrm{X}$-ray gas (as we infer), or even smaller, because magnetic pressure takes over as the density increases. In this case, one would again expect that the density in the X-ray gas is at least an order of magnitude higher than $n_{e} \sim 1 \mathrm{~cm}^{-3}$, and the filling factor correspondingly small.

\subsubsection{Feedback and the Energy Budget}

The thermal energy $E_{\text {th }}$ for most regions ranges between $\sim 10^{53}$ and $\sim 10^{55} \mathrm{erg}$. The large volumes of the cross-cone (regions 3 and 7) and outer bicone (6 and 13) imply that these extended regions can dominate energetically if the collisional component is real. Their inclusion increases the total $E_{\text {th }}$ by a factor of $\sim 13$, from $7.8 \times 10^{54}$ to $1.04 \times 10^{56} \mathrm{erg}$. Our assumptions of region thickness also remain major sources of uncertainty in these calculations; if the hot gas occupies a thin "skin" immediately above the galactic plane (as might be the case for wind-disk interactions beyond the S-shaped arms), then $E_{\text {th }}$ may become much smaller.

Regardless, our best estimates of $t_{\text {cool }}$ are at least factor of a few larger than $t_{\text {cross. }}$. In some cases (regions 1, 7, 11, and 13) the lower bound of $t_{\text {cool }}$ is unconstrained due to uncertainty in the flux. As in Paggi et al. (2012), the local sound speed $c_{s}$ is comparable to the shock velocity $\left(v_{\mathrm{sh}} \sim 100[\mathrm{kT} / 0.013 \mathrm{keV}]^{1 / 2}\right.$, as per Raga et al. 2002), which gives us $t_{\text {cross }}$. More sensitive observations (currently in progress) are necessary to improve measurements of $E_{\mathrm{th}}$ and $t_{\mathrm{cool}}$ at Chandra resolution. But these results suggest that no additional heating sources are required for most regions, and possibly all regions. The highest-velocity gas measured by HST (Fischer et al. 2013) and Gemini (Finlez et al. 2018) exceeds the local sound speed in these regions, so shocks must occur. These should also be fast enough (FWHM $\gtrsim 1000 \mathrm{~km} \mathrm{~s}^{-1}$ ) to generate X-rays.

The kinetic power $L_{K}$ should exceed $E_{\text {th }} / t_{\text {cross. }}$. The total for all regions is therefore $L_{K} \gtrsim 3.6 \times 10^{42} \mathrm{erg} \mathrm{s}^{-1}$. The bolometric luminosity $L_{\text {bol }} \sim 8 \times 10^{45} \mathrm{erg} \mathrm{s}^{-1}$ Koss et al. (2015), so $L_{K} / L_{\text {bol }} \sim 0.5 \%$. This is comparable to the $\sim 0.5$ efficiency suggested by the Hopkins \& Elvis (2010) two-stage feedback model, but is close enough that Hopkins \& Elvis (2010) may be relevant. Finlez et al. (2018) suggest that the NGC 3393 jet is launched into the galactic disk. If so, the pressure difference between the extended X-ray gas and radio jets suggests that the jet is relatively young and may continue to burrow through the circumnuclear ISM. But models of jet-disk interaction (e.g., Mukherjee et al. 2018) indicate that a jet in such a scenario may be redirected, ablating and expelling the circumnuclear ISM away from the disk and along the path of least resistance. Deeper radio observations will help determine whether the jets are escaping or remain trapped by the ISM, as with, e.g., NGC 404 (Nyland et al. 2017), and some AGN with evidence for recent accretion mode switching (Keel et al. 2015, 2017; Sartori et al. 2016).

\section{Conclusion}

Using spatially resolved X-ray spectroscopy of the ENLR of the Sy2 NGC 3393, we see the impact of a young radio outflow in shaping the inner $\sim \mathrm{kpc}$ of the galaxy. Chandra provides a critical window to interpreting this picture independently of optical (as explored by Papers I and II, and recently Finlez et al. 2018). Subarcsecond resolution is necessary to resolve emission line structure, and suggests that unresolved spectroscopy can mischaracterize the physical properties of the circumnuclear ISM in such a case.

Comparing X-ray wavebands centered on O VII, O VIII, and Ne IX, we see clear differences in line morphology, with O VII roughly tracing the optical line-emitting gas (particularly [O III]) but with localized enhancements of Ne IX associated with radio outflows, as has been seen in, e.g., NGC 4151 (Wang et al. 2011a) and Mrk 573 (Paggi et al. 2012). Emission associated with Ne IX $(0.905 \mathrm{keV})$ likely contains contributions from intermediate-state iron emission associated with thermalshocked or shock-forming plasma, and appears to be associated either with shocks or possibly precursor material photoionized by strong shocks. We find that emission line measurements made by Koss et al. (2015) using Chandra gratings support a dominant role for collisional plasma in the X-ray emission. Regardless of the exact nature of the emission origin, the measured sound speed in the collisional plasma suggests (when compared with optical data) that shocks must occur, and the total inferred kinetic power of the outflows is sufficient to evacuate gas from the galaxy via the Hopkins \& Elvis (2010) two-stage feedback model.

Although the jet power is sufficient to remove the gas over the long term, the current situation revealed by $\mathrm{X}$-rays is complex. If the outflows are ablating and deforming the circumnuclear ISM in the disk plane (see also Finlez et al. 2018), then they may be redirected, escaping perpendicular to 
the plane as the jets continue to burrow outwards. In addition to cross-cone photoionization (as from a "leaky torus" seen in other places, e.g., Paggi et al. 2012; Fabbiano et al. 2018a), there is evidence for a cocoon of cooler thermal plasma in the cross-cone that may be partially absorbed as seen by Wang et al. (2011b) in NGC 4151. Alternately, confinement of the the equatorial outflow seen in Finlez et al. (2018) may contribute to the heating of this component. Such a cool component is not expected to be bright in X-rays, but may be confirmed via detection in UV via, e.g., O VI $\lambda 1031,1036$ with the $H S T$ Cosmic Origins Spectrograph (COS) in NGC 3393 and possibly other galaxies with equatorial outflows (Lena et al. 2015; Riffel et al. 2015; Couto et al. 2017).

A more direct comparison between available optical and ALMA data cubes would prove informative, as would more sensitive Chandra and broadband radio observations. Our ongoing Chandra Large Program will better constrain spectroscopic measurements of the collisional plasma at $\sim 0$ !" 25 resolution. Better sensitivity at Chandra resolution is critical to confidently measuring the energy in the collisional component, particularly at large $(1 \mathrm{kpc})$ radii, where a low-density ionized wind may be present above the disk. Accompanying deep Jansky Very Large Array (JVLA) observations will be sensitive to lower-density plasma in the outflows, which is no longer strongly interacting with the circumnuclear ISM.

Studies with Chandra make it clear that subarcsecond resolution is necessary to study sub-kpc AGN outflows even at relatively low redshifts (like NGC 3393) without spatial confusion. This groundwork with Chandra is essential to interpreting any observations made with the future ESA $\mathrm{X}$-ray mission Athena. Athena will provide powerful diagnostics of the complex plasma that we investigate here, thanks to a collecting area and energy resolution greatly superior to any observatory to date (Nandra et al. 2013), but its spatial resolution $\left(\sim \mathrm{few}^{\prime \prime}\right)$ will confuse multiple physically distinct AGN phenomena even at the very low redshifts. Lynx, currently proposed for NASA in the 2020 Decadal Survey, will enable imaging spectroscopy at Chandra-like resolution but with effective area and energy resolution orders of magnitude better than Chandra (Gaskin et al. 2018). Lynx is necessary to efficiently measure the spatially resolved multicomponent hot outflows from AGN, and will confidently distinguish collisional plasma from reprocessing of photoionizing radiation by the ISM even in faint ENLR systems.

W.P.M. thanks Travis Fischer, Steve Kraemer, and Vinay Kashyap for helpful and informative discussions. W.P.M. was supported by Chandra grants GO5-16101X, GO8-19096X, and GO8-19099X, as well as HST grants HST-GO-14271.009-A and HST-GO-15350.001-A. The work of J.W. was supported by the National Key R\&D Program of China (2016YFA0400702) and the National Science Foundation of China (11473021, 11522323).

Facilities: CXO, HST.

\section{ORCID iDs}

W. Peter Maksym (1) https://orcid.org/0000-0002-2203-7889 Giuseppina Fabbiano (1) https://orcid.org/0000-00023554-3318

Martin Elvis iㅜ https://orcid.org/0000-0001-5060-1398
Margarita Karovska (ํ) https://orcid.org/0000-0003-1769-9201

Alessandro Paggi (i) https://orcid.org/0000-0002-5646-2410

John Raymond (10 https://orcid.org/0000-0002-7868-1622

Junfeng Wang (i) https://orcid.org/0000-0003-4874-0369

Thaisa Storchi-Bergmann (1) https://orcid.org/0000-00031772-0023

Guido Risaliti @i https://orcid.org/0000-0002-3556-977X

\section{References}

Alonso-Herrero, A., Simpson, C., Ward, M. J., \& Wilson, A. S. 1998, ApJ, 495, 196

Arnaud, K. A. 1996, in ASP Conf. Ser. 101, Astronomical Data Analysis Software and Systems V, ed. G. H. Jacoby \& J. Barnes (San Francisco, CA: ASP), 17

Ashman, K. M., Bird, C. M., \& Zepf, S. E. 1994, AJ, 108, 2348

Bautista, M. A., \& Kallman, T. R. 2000, ApJ, 544, 581

Bianchi, S., Guainazzi, M., \& Chiaberge, M. 2006, A\&A, 448, 499

Bogdán, Á, Kraft, R. P., Evans, D. A., Andrade-Santos, F., \& Forman, W. R. 2017, ApJ, 848, 61

Burlon, D., Ajello, M., Greiner, J., et al. 2011, ApJ, 728, 58

Cash, W. 1979, ApJ, 228, 939

Cooke, A. J., Baldwin, J. A., Ferland, G. J., Netzer, H., \& Wilson, A. S. 2000, ApJS, 129, 517

Couto, G. S., Storchi-Bergmann, T., \& Schnorr-Müller, A. 2017, MNRAS, 469, 1573

Dekel, A., \& Silk, J. 1986, ApJ, 303, 39

de Vaucouleurs, G., de Vaucouleurs, A., Corwin, H. G., Jr., et al. 1991, Third Reference Catalogue of Bright Galaxies. Vol. I, II, III (New York: Springer)

Diaz, A. I., Prieto, M. A., \& Wamsteker, W. 1988, A\&A, 195, 53

Fabbiano, G., Elvis, M., Paggi, A., et al. 2017, ApJL, 842, L4

Fabbiano, G., Paggi, A., Karovska, M., et al. 2018a, ApJ, 855, 131

Fabbiano, G., Paggi, A., Karovska, M., et al. 2018b, ApJ, 865, 83

Fabbiano, G., Wang, J., Elvis, M., \& Risaliti, G. 2011, Natur, 477, 431

Fabian, A. C. 2012, ARA\&A, 50, 455

Ferland, G. J., Korista, K. T., Verner, D. A., et al. 1998, PASP, 110, 761

Finlez, C., Nagar, N. M., Storchi-Bergmann, T., et al. 2018, MNRAS, 479, 3892

Fischer, T. C., Crenshaw, D. M., Kraemer, S. B., \& Schmitt, H. R. 2013, ApJS, 209, 1

Fruscione, A., McDowell, J. C., Allen, G. E., et al. 2006, Proc. SPIE, 6270, 62701

Gaskin, J. A., Dominguez, A., Gelmis, K., et al. 2018, Proc. SPIE, 10699, $106990 \mathrm{~N}$

Gaspari, M., \& Sądowski, A. 2017, ApJ, 837, 149

Guainazzi, M., Matt, G., \& Perola, G. C. 2005, A\&A, 444, 119

Heckman, T. M., \& Best, P. N. 2014, ARA\&A, 52, 589

Hopkins, P. F., \& Elvis, M. 2010, MNRAS, 401, 7

Keel, W. C., Lintott, C. J., Maksym, W. P., et al. 2017, ApJ, 835, 256

Keel, W. C., Maksym, W. P., Bennert, V. N., et al. 2015, AJ, 149, 155

Kinkhabwala, A., Sako, M., Behar, E., et al. 2002, ApJ, 575, 732

Koss, M. J., Romero-Cañizales, C., Baronchelli, L., et al. 2015, ApJ, 807, 149

Läsker, R., Greene, J. E., Seth, A., et al. 2016, ApJ, 825, 3

Lena, D., Robinson, A., Storchi-Bergman, T., et al. 2015, ApJ, 806, 84

Levenson, N. A., Heckman, T. M., Krolik, J. H., Weaver, K. A., \& Życki, P. T. 2006, ApJ, 648, 111

Liedahl, D. A., Kahn, S. M., Osterheld, A. L., \& Goldstein, W. H. 1990, ApJL, 350, L37

Maiolino, R., Salvati, M., Bassani, L., et al. 1998, A\&A, 338, 781

Maksym, W. P., Fabbiano, G., Elvis, M., et al. 2016, ApJ, 829, 46

Maksym, W. P., Fabbiano, G., Elvis, M., et al. 2017, ApJ, 844, 69

Mukherjee, D., Bicknell, G. V., Sutherland, R., \& Wagner, A. 2016, MNRAS, 461, 967

Mukherjee, D., Bicknell, G. V., Wagner, A. Y., Sutherland, R. S., \& Silk, J. 2018, MNRAS, 479, 5544

Nandra, K., Barret, D., Barcons, X., et al. 2013, arXiv:1306.2307

Nyland, K., Davis, T. A., Nguyen, D. D., et al. 2017, ApJ, 845, 50

Paggi, A., Wang, J., Fabbiano, G., Elvis, M., \& Karovska, M. 2012, ApJ, 756, 39

Paggi, A., Fabbiano, G., Risaliti, G., et al. 2017, ApJ, 841, 44

Park, T., Kashyap, V. L., Siemiginowska, A., et al. 2006, ApJ, 652, 610

Porquet, D., \& Dubau, J. 2000, A\&AS, 143, 495

Raga, A. C., Noriega-Crespo, A., \& Velázquez, P. F. 2002, ApJL, 576, L149 
Ricci, C., Trakhtenbrot, B., Koss, M. J., et al. 2017, Natur, 549, 488

Riffel, R. A., Storchi-Bergmann, T., \& Riffel, R. 2015, MNRAS, 451, 3587

Sartori, L. F., Schawinski, K., Koss, M. J., et al. 2016, MNRAS, 457, 3629

Silk, J. 1997, ApJ, 481, 703

Smith, R. K., Brickhouse, N. S., Liedahl, D. A., \& Raymond, J. C. 2001, ApJL, 556, L91
Theureau, G., Bottinelli, L., Coudreau-Durand, N., et al. 1998, A\&AS, 130,333

Voit, G. M., Donahue, M., O’Shea, B. W., et al. 2015, ApJL, 803, L21

Wang, J., Fabbiano, G., Elvis, M., et al. 2011a, ApJ, 736, 62

Wang, J., Fabbiano, G., Elvis, M., et al. 2011c, ApJ, 742, 23

Wang, J., Fabbiano, G., Risaliti, G., et al. 2011b, ApJ, 729, 75 